\title{
SENSITIVITY ANALYSIS OF AN INVERSE PROBLEM FOR THE WAVE EQUATION WITH CAUSTICS
}

\author{
GANG BAO AND HAI ZHANG
}

\section{INTRODUCTION}

This paper is concerned with the sensitivity (or stability) of the inverse problem of recovering the velocity field in a domain from the boundary dynamic Dirichlet-toNeumann map (DDtN) in the wave equation. Let $\Omega$ be a bounded strictly convex smooth domain in $\mathbb{R}^{d}, d \geq 2$, with boundary $\Gamma$. Let $c(x)$ be a velocity field in $\Omega$ which characterizes the wave speed in the medium and let $T$ be a sufficiently large positive number. We consider the following wave equation system:

$$
\begin{aligned}
\frac{1}{c^{2}(x)} u_{t t}-\Delta u & =0, \quad(x, t) \in \mathbb{R}^{d} \times(0, T), \\
u(0, x)=u_{t}(0, x) & =0, \quad x \in \Omega, \\
u(x, t) & =f(x, t), \quad(x, t) \in \Gamma \times(0, T) .
\end{aligned}
$$

For each $f \in H_{0}^{1}([0, T] \times \Gamma)$, it is known that (see for instance [16]) there exists a unique solution $u \in C^{1}\left(0, T ; L^{2}(\Omega)\right) \bigcap C\left(0, T ; H^{1}(\Omega)\right)$, and furthermore $\frac{\partial u}{\partial \nu} \in$ $L^{2}([0, T] \times \Gamma)$, where $\nu$ is the unit outward normal to the boundary. The DDtN map $\Lambda_{c}$ is defined by

$$
\Lambda_{c}(f):=\left.\frac{\partial u}{\partial \nu}\right|_{[0, T] \times \Gamma} .
$$

The inverse problem is to recover the velocity function $c$ from the DDtN map $\Lambda_{c}$. The uniqueness of the inverse problem is solved by the boundary control method first introduced by Belishev in [7]. The method can also be used to solve the uniqueness for more general problems, for instance, the anisotropic medium case. We refer to [8, 10], 9], [15], 23] and the references therein for more discussions.

We are interested in the sensitivity question for the above inverse problem. Namely, we want to investigate how sensitive or stable is it to recover the velocity field from the DDtN map and characterize how a small change in the DDtN map affects the recovered velocity field.

The above inverse problem can be viewed as a special case of the problem of recovering a Riemannian metric on a Riemannian manifold. Indeed, it corresponds to the case when the metrics are restricted to the class of those which are conformal to the Euclidean one. The inverse problem of recovering a Riemannian metric has been extensively studied in the literature. The uniqueness is proved by Belishev

Received by the editors December 17, 2012 and, in revised form, July 20, 2013.

2010 Mathematics Subject Classification. Primary 35R30; Secondary 35S30.

The research was supported in part by the NSF grants DMS-0968360, DMS-1211292, the ONR grant N00014-12-1-0319, a Key Project of the Major Research Plan of NSFC (No. 91130004), and a special research grant from Zhejiang University. 
and Kurylev in [10] by using the boundary control method. However, as pointed out in 28, their approach is unlikely to give a stability estimate since it uses, in an essential way, a unique continuation property of the wave equation.

The first stability result on the determination of the metric from the DDtN map was given by Stefanov and Uhlmann in 25, where they proved conditional stability of Hölder type for metrics close enough to the Euclidean one in $C^{k}$ for $k \gg 1$ in three dimensions. Later, they extended the stability result to generic simple metrics 28. An important feature of their approach is to first derive a stability estimate of recovering the boundary distance function from the DDtN map and then apply existing results from the boundary rigidity problem in geometry. Their approach was extended by Montalto in [19] to study the more general problem of determining a metric, a co-vector and a potential simultaneously from the DDtN map, and a similar Hölder type conditional stability result was obtained. The stability of the inverse problem of determining the conformal factor to a fixed simple metric was studied by Bellassoued and Ferreira in [11. They proved the Hölder type conditional stability result for the case when the conformal factors are close to one. We comment that the result in [11] holds for all simple metrics. For other stability results on the related problems, we refer to the references in [19].

We emphasize that all of the above stability results deal with the case when the metrics are simple. To our best knowledge, no stability result is available in the general case when the metrics are not simple. This paper is devoted to the study of the general case when the metric induced by the velocity field is not simple. To avoid technical complications due to the boundary, we restrict our study to situations when the velocity fields are equal to one near the boundary. From this point of view, our results can be regarded as interior estimates. We refer to [28], [30] and the references therein for useful boundary estimates.

We now give a brief account of the approach and results in the paper. We first derive a sensitivity result of recovering the scattering relation from the DDtN map. Our result shows that two scattering relations must be identical if the two corresponding DDtN maps are sufficiently close in some suitable norm. Equivalently, any arbitrarily small change in the scattering relation can imply a certain change in the DDtN map. To our best knowledge, this seems to be the first sensitivity result for the inverse problem in the non-simple metric case. Moreover, our result is fundamentally different from those in the literature where Lipschitz, Hölder or logarithmic estimates are derived; see, for example, 6], 1], 33, 25], [26], 27], 28], 19] and [14. This is the reason the term "sensitivity analysis" is used instead of "stability estimates" throughout the paper. We remark that when the geometry induced by the velocity field is simple, the scattering relation is equivalent to the boundary distance function. In that case, a Hölder type interior stability estimate for recovering the boundary distance function from the DDtN map has been established in 28. Compared to the type of result, our result is much stronger. As a consequence, a stronger result than the Hölder type stability may be proved for the results in [28, [11] and [19. Our approach is based on Gaussian beam solutions to the wave equation, which are capable of dealing with caustics, major obstacles to the construction of classic geometric-optics solutions. We refer to [22] and [15] for more discussions on Gaussian beams and its applications. 
Once the above sensitivity result of recovering the scattering relation from its associated DDtN map is established, the sensitivity of the inverse problem of recovering the velocity field from its induced DDtN map is reduced to the uniqueness issue of recovering the velocity field from its induced scattering relation. This is a special case of the lens rigidity problem in geometry when the metrics are restricted to a conformal class (see [18 and 32]). With regard to the lens rigidity problem, Stefanov and Uhlmann ([32]) proved uniqueness up to diffeomorphisms fixing the boundary for metrics a priori close to a generic "regular" one. There "regular" means that there is a set of geodesics without conjugate points whose conormal bundles can cover the contangent bundle of the underlying manifold. Their approach is based on investigating the X-ray transform which is obtained from linearizing the boundary distance function with respect to the metric. The lens rigidity problem remains open for more general non-simple metrics.

In this paper, motivated by 35] which studies the geodesic X-ray transform with fold caustics, we introduce the "fold-regular" class (see Subsection 3.4 for definition) of velocity fields (or equivalently conformal metrics) which generalizes the above "regular" class, and study an linearized problem of a variant of the lens rigidity problem when the metrics are restricted to those conformal to the Euclidean one. Our approach is based on linearizing the Hamiltonian flow with respect to the velocity field. This gives us the advantage of handling geodesics with caustics in comparison to the approach by using the boundary distance function. More specifically, we linearize the operator which maps $c$ to $\left.\mathcal{H}_{c}^{T}\right|_{S^{*} \mathbb{R}^{d}}$ and obtain a geodesic X-ray transform operator $\mathfrak{I}_{c}$ with matrix-valued weight. We study the inverse problem of recovering a vector-valued function $f$ from its X-ray transform $\mathfrak{I}_{c} f$. For a fixed interior point $x$, we use a carefully selected set of geodesics whose conormal bundle can cover the cotangent space $T_{x}^{*} \mathbb{R}^{d}$ to recover the singularity of $f$ at $x$. We allow fold caustics along these geodesics, but require that these caustics contribute to a smoother term in the transform than $x$ itself. It is still an open problem to show that such a set of geodesics exists generically for a general velocity field with caustics. But we draw evidence from the classification result on caustics and regularity theory of Fourier Integral Operators (FIOs) to show that it is the case under some natural assumptions in the dimensions equal or greater than three. We call the interior point with the above set of geodesics "foldregular". See Section 6.3 for discussions on the new concept "fold-regular". A local stability estimate is derived near a fold-regular point. We think that this local stability estimate and the approach presented here may hold the key for analyzing the stability of the lens rigidity problem with general non-simple metrics, which is completely open at present. Initial progress has been made along this direction in 36.

Finally, we combine the stability result on the X-ray transform and the sensitivity result on recovering the scattering relation from the DDtN map to obtain a sensitivity result for the inverse problem.

The paper is organized as follows. In Section 2, we present some preliminaries. In Section 3, we introduce the main results in the paper. Section 4 is devoted to the construction of Gaussian beam solutions to the wave equation. The Gaussian beam solutions are used in Section 5 to prove the sensitivity result of determining the scattering relation from the DDtN map. In Section 6, we discuss the concept "fold-regular" and prove a local stability estimate for the geodesic X-ray transform 
$\mathfrak{I}_{c}$. In Section 7 , we prove the sensitivity result of recovering the velocity field from the DDtN map.

Throughout, we use the following conventions:

(1) Let $f$ and $g$ be two elements in a Hilbert space, then $\langle f, g\rangle$ stands for their inner product.

(2) Let $M_{1}$ and $M_{2}$ be two matrices (including vectors which can be regarded as single column or single row matrices), then the product of $M_{1}$ and $M_{2}$ is denoted by $M_{1} \cdot M_{2}$. Sometimes, the dot is omitted for simplicity.

(3) Let $M$ be a matrix, then $M^{\dagger}$ stands for its transpose. The same applies when $M$ is a linear operator. If $M$ is real and symmetric and $C$ a real number, then $M \geq C$ means that the matrix $M-C \cdot I d$ is symmetric and positive definite. If $M$ is a complex matrix, then we use $\Re M$ for its real part and $\Im M$ for its imaginary part.

(4) Let $U$ and $V$ be two open sets in a metric space, then $U \Subset V$ means that the closure of $U$, denoted by $\bar{U}$, is compact and is a subset of $V$.

(5) Let $C_{1}$ and $C_{2}$ be two positive numbers, then $C_{1} \lesssim C_{2}$ means that $C_{1} \leq$ $C \cdot C_{2}$ for some constant $C>0$ independent of $C_{1}$ and $C_{2}$.

\section{Preliminaries}

In this section, we introduce some notations and definitions. Let $\Omega$ be a strictly convex smooth domain in $\mathbb{R}^{d}$ with boundary $\Gamma$. Let $c$ be a smooth velocity field defined in $\Omega$ which is equal to one near the boundary. Then $c$ has a natural extension to $\mathbb{R}^{d}$. Throughout the paper, we always use the natural coordinate system of the cotangent bundle $T^{*} \mathbb{R}^{d}$ in which we write $(x, \xi)$ for the co-vector $\xi_{j} d x^{j}$ in $T_{x}^{*} \mathbb{R}^{d}$. For ease of notation, we also use $\xi$ for the co-vector $\xi_{j} d x^{j}$. The meaning of $\xi$ should be clear from the context. The velocity field $c$ introduces a Hamiltonian function $H_{c}(x, \xi)=\frac{1}{2} c^{2}(x)\left(\xi_{1}^{2}+\cdots \xi_{d}^{2}\right)$ to $T^{*} \mathbb{R}^{d}$. It also defines a norm to each cotangent space $T_{x}^{*} \mathbb{R}^{d}$ by

$$
|\xi|=c(x) \sqrt{\xi_{1}^{2}+\cdots \xi_{d}^{2}}, \quad \text { for } \xi \in T_{x}^{*} \mathbb{R}^{d} .
$$

Denote the corresponding Hamiltonian flow by $\mathcal{H}_{c}^{t}$; i.e., for each $\left(x_{0}, \xi_{0}\right) \in T^{*} \mathbb{R}^{d}$, $\mathcal{H}_{c}^{t}\left(x_{0}, \xi_{0}\right)=\left(x\left(t, x_{0}, \xi_{0}\right), \xi\left(t, x_{0}, \xi_{0}\right)\right)$ solves the following equations:

$$
\begin{aligned}
& \dot{x}=\frac{\partial H_{c}}{\partial \xi}=c^{2} \cdot \xi, \quad x(0)=x_{0}, \\
& \dot{\xi}=-\frac{\partial H_{c}}{\partial x}=-\frac{1}{2} \nabla c^{2} \cdot\left(\xi_{1}^{2}+\cdots \xi_{d}^{2}\right), \quad \xi(0)=\xi_{0} .
\end{aligned}
$$

We call $\left(x\left(\cdot, x_{0}, \xi_{0}\right), \xi\left(\cdot, x_{0}, \xi_{0}\right)\right)$ the bicharacteristic curve emanating from $\left(x_{0}, \xi_{0}\right)$ and $x\left(\cdot, x_{0}, \xi_{0}\right)$ the geodesic. By the assumptions on $c$, the flow $\mathcal{H}_{c}^{t}$ is defined for all $t \in \mathbb{R}$. Note that the flow $\mathcal{H}_{c}^{t}$ is also well-defined on the cosphere bundle $S^{*} \mathbb{R}^{d}=\left\{(x, \xi): x \in \mathbb{R}^{d},|\xi|=1\right\}$.

We say that a velocity field $c$ is non-trapping in $\Omega$ for time $T>0$ if the following condition is satisfied:

$$
\mathcal{H}_{c}^{T}\left(S^{*} \Omega\right) \bigcap S^{*} \Omega=\emptyset .
$$


Denote

$$
\begin{aligned}
& S_{+}^{*} \Gamma=\{(x, \xi): x \in \Gamma,|\xi|=1,\langle\xi, \nu(x)\rangle>0\} \\
& S_{-}^{*} \Gamma=\{(x, \xi): x \in \Gamma,|\xi|=1,\langle\xi, \nu(x)\rangle<0\} .
\end{aligned}
$$

Assume that the velocity field $c$ is non-trapping in $\Omega$ for time $T$; we now define the scattering relation $\mathfrak{S}_{c}: S_{-}^{*} \Gamma \rightarrow S_{+}^{*} \Gamma$. For each $\left(x_{0}, \xi_{0}\right) \in S_{-}^{*} \Gamma$, let $l\left(x_{0}, \xi_{0}\right)$ be the first moment that the geodesic $x\left(\cdot, x_{0}, \xi_{0}\right)$ hits the boundary $\Gamma$. Define

$$
\mathfrak{S}_{c}\left(x_{0}, \xi_{0}\right)=\mathcal{H}_{c}^{l\left(x_{0}, \xi_{0}\right)}\left(x_{0}, \xi_{0}\right) .
$$

For future reference, we define $l_{-}: S^{*} \Omega \rightarrow(-\infty, 0]$ by letting $l_{-}(x, \xi)$ be the first negative moment that the bicharacteristic curve $\mathcal{H}^{t}(x, \xi)$ hits the boundary $S_{-}^{*} \Gamma$ and $\tau: S^{*} \Omega \rightarrow S_{-}^{*} \Gamma$ by

$$
\tau(x, \xi)=\mathcal{H}^{l_{-}(x, \xi)}(x, \xi) .
$$

We remark that $l_{-}(\cdot)$ and $\tau(\cdot)$ are well-defined by the assumption (6).

We now introduce the class of admissible velocity fields that are considered in the paper.

Definition 2.1. Let $M_{0}, \epsilon_{0}$ and $T$ be positive numbers. A velocity field $c$ is said to belong to the admissible class $\mathfrak{A}\left(M_{0}, \epsilon_{0}, \Omega, T\right)$ if and only if the following three conditions are satisfied:

(1) $c \in C^{3}\left(\mathbb{R}^{d}\right), 0<\frac{1}{M_{0}} \leq c \leq M_{0}$, and $\|c\|_{C^{3}\left(\mathbb{R}^{d}\right)} \leq M_{0}$;

(2) the support of $c-1$ is contained in the set $\Omega_{\epsilon_{0}}=:\left\{x \in \Omega: \operatorname{dist}(x, \Gamma)>\epsilon_{0}\right\}$;

(3) the Hamiltonian $H_{c}$ is non-trapping in $\Omega$ for time $T$.

By Condition (2) above and the assumption that $\Omega$ is bounded and convex, it is easy to verify that any ray starting in the domain $\Omega_{\epsilon_{0}}$ intersects $\Gamma$ transversely. Moreover, there exist two small positive constants $\epsilon^{*}$ and $\epsilon_{1}$, both depending on $\epsilon_{0}$, such that for any $\left(x_{0}, \xi_{0}\right) \in S_{-}^{*} \Gamma$, if $\left\{\mathcal{H}^{t}\left(x_{0}, \xi_{0}\right): t \in\left(0, l\left(x_{0}, \xi_{0}\right)\right)\right\} \cap S^{*} \Omega_{\epsilon_{0}} \neq \emptyset$, then

$$
\begin{aligned}
\left\langle\xi_{0}, \nu\left(x_{0}\right)\right\rangle & \leq-\epsilon^{*}, \\
\left\langle\xi_{1}, \nu\left(x_{1}\right)\right\rangle & \geq \epsilon^{*}, \\
l\left(x_{0}, \xi_{0}\right) & \geq \epsilon_{1},
\end{aligned}
$$

where $\left(x_{1}, \xi_{1}\right)=\mathfrak{S}_{c}\left(x_{0}, \xi_{0}\right)$.

Finally, we remark that we set up the discussion in the paper in the cotangent space $T^{*} \mathbb{R}^{d}$. But one can also set up the discussion in the tangent space $T \mathbb{R}^{d}$; see, for instance, 24, 32. The equivalence of the two setups can be seen from the procedure of "raising and lowing indices" in the Riemannian geometry. We choose the cotangent setup mainly because of the following three reasons. First, it is more natural to the construction of Gaussian beams. Second, the classification result of singular Lagrangian maps is more complete than that of singular exponential maps in the literature, though these two problems are equivalent in the Riemannian manifold. Finally, it is more natural to study caustics in the cotangent space. 


\section{Statement of the main Results}

3.1. Sensitivity of recovering the scattering relation from the DDtN map. It is known that the DDtN map $\Lambda_{c}$ determines the scattering relation $\mathfrak{S}_{c}$ uniquely [21. We show that the following sensitivity result of recovering the scattering relation from the DDtN map holds. The proof is given in Section 5 .

Theorem 3.1. Let $c$ and $\tilde{c}$ be two velocity fields in the class $\mathfrak{A}\left(\epsilon_{0}, \Omega, M_{0}, T\right)$. Then there exists a constant $\delta>0$ such that

$$
\begin{aligned}
& \mathfrak{S}_{\tilde{c}}=\mathfrak{S}_{c} \\
& \text { if }\left\|\Lambda_{\tilde{c}}-\Lambda_{c}\right\|_{H_{0}^{1}\left[0,3 \epsilon_{1} / 4\right] \times \Gamma \rightarrow L^{2}\left(\left[0, T+\epsilon_{1}\right] \times \Gamma\right)} \leq \delta \text {. }
\end{aligned}
$$

Remark 3.1. The same result holds when the velocity fields are replaced by symmetric positive definite matrices.

3.2. Linearization of the operator which maps velocity fields to Hamiltonian flows. We begin with the following observation.

Lemma 3.1. Let $c$ and $\tilde{c}$ be two velocity fields in the class $\mathfrak{A}\left(\epsilon_{0}, \Omega, M_{0}, T\right)$, then $\mathfrak{S}_{c}=\mathfrak{S}_{\tilde{c}}$ if and only if $\left.\mathcal{H}_{c}^{T}\right|_{S_{-}^{*} \Gamma}=\left.\mathcal{H}_{\tilde{c}}^{T}\right|_{S_{-}^{*} \Gamma}$.

The above lemma shows the equivalence of the Hamiltonian flow and the scattering relation. The next lemma shows that $\mathcal{H}_{c}^{t}$ satisfies an equivalent ordinary differential equation (ODE) system in $S^{*} \mathbb{R}^{d}$.

Lemma 3.2. Let $\left(x_{0}, \xi_{0}\right) \in S^{*} \mathbb{R}^{d}=\left\{(x, \xi) \in \mathbb{R}^{2 d}:|\xi|=1\right\}$, and let $(x(t), \xi(t))=$ $\mathcal{H}_{c}^{t}\left(x_{0}, \xi_{0}\right)$, then $(x(t), \xi(t))$ satisfies the following ODE system:

$$
\begin{aligned}
& \dot{x}=\frac{\xi}{\xi_{1}^{2}+\cdots+\xi_{d}^{2}}, \\
& \dot{\xi}=b(x),
\end{aligned}
$$

where $b(x)=-\frac{1}{2} \nabla \ln c^{2}$. Conversely, if $(x(t), \xi(t)) \in S^{*} \mathbb{R}^{d}$ satisfies the ODE system (10) - (11), then $(x(t), \xi(t))=\mathcal{H}_{c}^{t}\left(x_{0}, \xi_{0}\right)$.

We next linearize the operator which maps each velocity field to its induced Hamiltonian flow restricted to the cosphere bundle. Let $c$ be a fixed smooth background velocity field. Denote the perturbed velocity field and Hamiltonian flow at time $T$ as $\tilde{c}^{2}=c^{2}+\delta c^{2}$ and $\mathcal{H}_{\tilde{c}}^{T}=\mathcal{H}_{c}^{T}+\delta \mathcal{H}_{c}^{T}$, respectively. Denote also that $\delta b=-\frac{1}{2} \nabla\left(\ln \tilde{c}^{2}-\ln c^{2}\right)$ and

$$
A(x, \xi)=\left(\begin{array}{cc}
0 & \frac{\partial}{\partial \xi}\left(\frac{\xi}{\xi_{1}^{2}+\cdots+\xi_{d}^{2}}\right) \\
\frac{\partial b}{\partial x} & 0
\end{array}\right) .
$$

For each $\left(x_{0}, \xi_{0}\right) \in S_{-}^{*} \Gamma$, let $\Upsilon\left(t, x_{0}, \xi_{0}\right)$ be the solution of the following ODE system:

$$
\dot{\Upsilon}(t)=-\Upsilon(t) A\left(\mathcal{H}_{c}^{t}\left(x_{0}, \xi_{0}\right)\right), \quad \Upsilon(0)=I d .
$$

By the results in Appendix 8.1 we have

where

$$
\delta \mathcal{H}_{c}^{T}=\frac{\delta \mathcal{H}_{c}^{T}}{\delta b}(\delta b)+r(\delta b)
$$

$$
\frac{\delta \mathcal{H}_{c}^{T}}{\delta b}(\delta b)\left(x_{0}, \xi_{0}\right)=\int_{0}^{T} \Upsilon^{-1}\left(T, x_{0}, \xi_{0}\right) \cdot \Upsilon\left(s, x_{0}, \xi_{0}\right)\left(\begin{array}{c}
0 \\
\delta b\left(x\left(s, x_{0}, \xi_{0}\right)\right)
\end{array}\right) d s
$$


and $\|r(\delta b)\|_{L^{\infty}} \leq C\|\delta b\|_{C^{1}}^{2}$ for some constant $C>0$ depending only on $\|c\|_{C^{3}\left(\mathbb{R}^{d}\right)}$.

Formula (12) motivates us to define the following geodesic X-ray transform operator:

$$
\mathfrak{I}_{c}(f)\left(x_{0}, \xi_{0}\right)=\int_{0}^{T} \Upsilon\left(s, x_{0}, \xi_{0}\right) f\left(x\left(s, x_{0}, \xi_{0}\right)\right) d s, \quad f \in \mathcal{E}^{\prime}\left(\Omega, \mathbb{R}^{2 d}\right) .
$$

Then $\frac{\delta \mathcal{H}_{c}^{T}}{\delta b}(\delta b)\left(x_{0}, \xi_{0}\right)=\Upsilon^{-1}\left(T, x_{0}, \xi_{0}\right) \cdot \mathfrak{I}_{c}(f)\left(x_{0}, \xi_{0}\right)$ with

$$
f=\left(\begin{array}{c}
0 \\
\frac{1}{2} \nabla\left(\ln c^{2}-\ln \tilde{c}^{2}\right)
\end{array}\right) .
$$

We associate each $(x, \xi) \in S^{*} \Omega$ with a matrix $\Phi(x, \xi)$. Let $\left(x_{0}, \xi_{0}\right)=\tau(x, \xi)=$ $\mathcal{H}_{c}^{l_{-}(x, \xi)}(x, \xi)$. We then define

$$
\Phi(x, \xi)=\Upsilon\left(-l_{-}(x, \xi), \tau(x, \xi)\right) .
$$

By definition, it is clear that

$$
\Phi\left(\mathcal{H}_{c}^{s}\left(x_{0}, \xi_{0}\right)\right)=\Upsilon\left(s, x_{0}, \xi_{0}\right)
$$

for all $s \in \mathbb{R}_{+}$such that $\mathcal{H}_{c}^{s}\left(x_{0}, \xi_{0}\right) \in S^{*} \Omega$. Note that $f\left(x\left(s, x_{0}, \xi_{0}\right)\right)=0$ if $s \geq$ $l\left(x_{0}, \xi_{0}\right)$ for $f \in \mathcal{E}^{\prime}\left(\Omega, \mathbb{R}^{2 d}\right)$. We can rewrite the X-ray transform operator $\mathfrak{I}_{c}$ (13) in the following standard form:

$$
\begin{aligned}
\mathfrak{I}_{c} f\left(x_{0}, \xi_{0}\right) & =\int_{0}^{l\left(x_{0}, \xi_{0}\right)} \Upsilon\left(s, x_{0}, \xi_{0}\right) f\left(x\left(s, x_{0}, \xi_{0}\right)\right) d s, \\
& =\int_{0}^{l\left(x_{0}, \xi_{0}\right)} \Phi\left(\mathcal{H}_{c}^{s}\left(x_{0}, \xi_{0}\right)\right) f\left(\pi\left(\mathcal{H}_{c}^{s}\left(x_{0}, \xi_{0}\right)\right)\right) d s .
\end{aligned}
$$

Remark 3.2. Formula (15) is derived in the coordinate of $T^{*} \mathbb{R}^{d}$. Hence it may not be geometrically invariant.

Lemma 3.3. Assume that $\mathfrak{S}_{c}=\mathfrak{S}_{\tilde{c}}$; let $f$ be defined as in (14), then

$$
\left\|\mathfrak{I}_{c} f\right\|_{L^{\infty}} \lesssim\|f\|_{C^{1}(\Omega)}^{2} .
$$

Proof: First, using $\mathfrak{S}_{c}=\mathfrak{S}_{\tilde{c}}$ and Lemma 3.1, we have

$$
\left.\delta \mathcal{H}_{c}^{T}\right|_{S_{-}^{*} \Gamma}=\left.\mathcal{H}_{\tilde{c}}^{T}\right|_{S_{-}^{*} \Gamma}-\left.\mathcal{H}_{c}^{T}\right|_{S_{-}^{*} \Gamma}=0 .
$$

Next, by the proceeding discussion, we have

$$
\delta \mathcal{H}_{c}^{T}=\frac{\delta \mathcal{H}_{c}^{T}}{\delta b}(\delta b)+r(\delta b),
$$

where the term $r$ satisfies the following inequality:

$$
\|r(\delta b)\|_{L^{\infty}} \leq C\|\delta b\|_{C^{1}}^{2}
$$

for some constant $C>0$ depending only on $\|c\|_{C^{3}\left(\mathbb{R}^{d}\right)}$. Combining (16)-(18), we see that

Finally, using the equality

$$
\left\|\frac{\delta \mathcal{H}_{c}^{T}}{\delta b}(\delta b)\right\|_{L^{\infty}}=\|r(\delta b)\|_{L^{\infty}} \lesssim\|\delta b\|_{C^{1}}^{2}=\|f\|_{C^{1}}^{2} .
$$

$$
\mathfrak{I}_{c}(f)\left(x_{0}, \xi_{0}\right)=\Upsilon\left(T, x_{0}, \xi_{0}\right) \cdot \frac{\delta \mathcal{H}_{c}^{T}}{\delta b}(\delta b)\left(x_{0}, \xi_{0}\right)
$$


and the fact that the matrix-valued function $\Upsilon(T, \cdot, \cdot)$ is a smooth function determined by the background velocity field $c$, we get the desired conclusion immediately.

3.3. Fold-regular points and local stability for geodesic X-ray transform. We consider the stability estimate of the operator $\mathfrak{I}_{c}$. For simplicity, we drop the subscript $c$. Define $\beta: T^{*} \mathbb{R}^{d} \backslash\left\{(x, 0): x \in \mathbb{R}^{d}\right\} \rightarrow S^{*} \mathbb{R}^{d}$ by

$$
\beta(x, \xi)=\left(x, \frac{\xi}{|\xi|}\right) .
$$

Let $\pi: T^{*} \mathbb{R}^{d} \rightarrow \mathbb{R}^{d}$ be the natural projection onto the base space. We define $\phi: T^{*} \mathbb{R}^{d} \rightarrow \mathbb{R}^{d}$ by

$$
\phi(x, \xi)=\pi \circ \mathcal{H}^{t=1}(x, \xi), \quad(x, \xi) \in T^{*} \mathbb{R}^{d} .
$$

We remark that $\phi$ defined above is equivalent to the exponential map in the Riemannian manifold.

The following result about the normal operator $\mathfrak{N}=\mathfrak{I}^{\dagger} \mathfrak{I}$ is well-known.

Lemma 3.4. The normal operator $\mathfrak{N}: L^{2}\left(\Omega, \mathbb{R}^{2 d}\right) \rightarrow L^{2}\left(\Omega, \mathbb{R}^{2 d}\right)$ is bounded and has the following representation:

$$
\mathfrak{N} f(x)=\int_{T_{x}^{*} \Omega} W(x, \xi) f(\phi(x, \xi)) d \sigma_{x}(\xi), \quad f \in L^{2}\left(\Omega, \mathbb{R}^{2 d}\right),
$$

where $d \sigma_{x}$ denotes the measure in the space $T_{x}^{*} \mathbb{R}^{d}$ induced by the velocity field $c$; i.e., $d \sigma_{x}(\xi)=c(x)^{d} d \xi$, and $W$ is defined as

$$
W(x, \xi)=\frac{1}{|\xi|^{d-1}}\left\{\Phi^{\dagger} \circ \beta(x, \xi) \cdot \Phi \circ \beta \circ \mathcal{H}(x, \xi)+\Phi^{\dagger} \circ \beta(x,-\xi) \cdot \Phi \circ \beta \circ \mathcal{H}^{-1}(x,-\xi)\right\} .
$$

Proof. See [35] or 26].

We see from (20) that the local property of the normal operator $\mathfrak{N}$ restricted to a small neighborhood of $x \in \Omega$ is determined by the Lagrangian map $\phi(x, \cdot)$ : $T_{x}^{*} \mathbb{R}^{d} \rightarrow \mathbb{R}^{d}$. When the map is a diffeomorphism, it is known that the operator $\mathfrak{N}$ near $x$ is a pseudo-differential operator $(\Psi D O)$. However, in the general case, the map may not be a diffeomorphism and may have singular points which are called caustic vectors. The value of the map at caustic vectors are called caustics. When caustics occur, the Schwartz kernel of the operator $\mathfrak{N}$ has two singularities, one is from the diagonal which contributes to a $\Psi D O \mathfrak{N}_{1}$, and the other is from the caustics which contributes to a singular integral operator $\mathfrak{N}_{2}$. The property of $\mathfrak{N}_{2}$ depends on the type of caustics. The case for fold caustics is investigated in 35, where it is shown that fold caustics contribute a Fourier Integral Operator (FIO) to $\mathfrak{N}_{2}$. Little is known for caustics of other types. Here we recall the following definition of fold caustics.

Definition 3.1. Let $f: \mathbb{R}^{n} \rightarrow \mathbb{R}^{n}$ be a germ of $C^{\infty}$ map at $x_{0}$, then $x_{0}$ is said to be a fold vector and $f\left(x_{0}\right)$ a fold caustic if the following two conditions are satisfied:

(1) the rank of $d f$ at $x_{0}$ equals to $n-1$ and det $d f$ vanishes of order 1 at $x_{0}$;

(2) the kernel of the matrix $d f\left(x_{0}\right)$ is transversal to the manifold $\{x: \operatorname{det} d f(x)=$ $0\}$ at $x_{0}$. 
We now introduce the following concept of "operator germ" to characterize the contribution of an infinitesimal neighborhood of a caustic or a regular point to the normal operator $\mathfrak{N}$.

Definition 3.2. For each $\xi \in T_{x}^{*} \mathbb{R}^{d} \backslash 0$, the operator germ $\mathfrak{N}_{\xi}$ is defined to be the equivalent class of operators in the following form:

$$
\mathfrak{N}_{\xi} f(y)=\int_{T_{y}^{*} \Omega} W(y, \eta) f(\phi(y, \eta)) \chi(y, \eta) d \sigma_{y}(\eta),
$$

where $\chi$ is a smooth function supported in a small neighborhood of $(x, \xi)$ in $\mathbb{R}^{2 d}$. Two operators with $\chi_{1}$ and $\chi_{2}$ are said to be equivalent if there exists a neighborhood $B(x, \xi)$ of $(x, \xi)$ such that $\chi_{1}=\chi_{2} \cdot \chi_{3}$ for some $\chi_{3} \in C_{0}^{\infty}(B(x, \xi))$ with $\chi_{3}(x, \xi) \neq 0$.

The operator germ $\mathfrak{N}_{\xi}$ is said to have certain property if there exists a neighborhood $B(x, \xi)$ of $(x, \xi)$ in $T^{*} \mathbb{R}^{d}$ such that the property holds for all operators of the form (22) with $\chi \in C_{0}^{\infty}(B(x, \xi))$.

Properties of the above defined operator germ will be given in Section 6.1 .

We note from the preceding discussion that it is complicated to analyze the full operator $\mathfrak{N}$ which contains information from all geodesics. However, for a given interior point $x$, to recover $f$ or the singularity of $f$ at $x$ from its geodesic transform, we need only to select a set of geodesics whose conormal bundle can cover the cotangent space $T_{x}^{*} \mathbb{R}^{d}$. Caustics may be allowed along these geodesics as long as they are of the simplest type, i.e., fold type so that we can analyze their contributions. This idea can be carried out by introducing a cut-off function for the set of geodesics as we do now. We remark that this idea is motivated by the work [30. For any $\alpha \in C_{0}^{\infty}\left(S_{-}^{*} \Gamma\right)$, we define

$$
\mathfrak{I}_{\alpha} f\left(x_{0}, \xi_{0}\right)=\alpha\left(x_{0}, \xi_{0}\right) \int_{0}^{l\left(x_{0}, \xi_{0}\right)} \Phi\left(\mathcal{H}_{c}^{s}\left(x_{0}, \xi_{0}\right)\right) f\left(\pi\left(\mathcal{H}_{c}^{s}\left(x_{0}, \xi_{0}\right)\right)\right) d s,
$$

where $\left(x_{0}, \xi_{0}\right) \in S_{-}^{*} \Gamma$. Let $\alpha^{\sharp}$ be the unique lift of $\alpha$ to $S^{*} \Omega$ which is constant along bicharacteristic curves, i.e., $\alpha^{\sharp}(x, \xi)=\alpha \circ \tau(x, \xi)$ for $(x, \xi) \in S^{*} \Omega$. Then $\alpha^{\sharp}$ is smooth in $S^{*} \Omega$ and we have

$$
\Im_{\alpha} f\left(x_{0}, \xi_{0}\right)=\int_{0}^{l\left(x_{0}, \xi_{0}\right)}\left(\alpha^{\sharp} \cdot \Phi\right)\left(\mathcal{H}_{c}^{s}\left(x_{0}, \xi_{0}\right)\right) f\left(\pi\left(\mathcal{H}_{c}^{s}\left(x_{0}, \xi_{0}\right)\right)\right) d s .
$$

With the original weight $\Phi$ being replaced by the new one $\alpha^{\sharp} . \Phi$, we similarly can define $\mathfrak{N}_{\alpha}$. In fact, it is easy to check that $\mathfrak{N}_{\alpha}$ is defined as in (21) with $W$ being replaced by

$$
\begin{aligned}
W_{\alpha}(x, \xi)= & \frac{1}{|\xi|^{d-1}}|\alpha \circ \tau \circ \beta(x, \xi)|^{2} \Phi^{\dagger} \circ \beta(x, \xi) \cdot \Phi \circ \beta \circ \mathcal{H}(x, \xi) \\
& +\frac{1}{|\xi|^{d-1}}|\alpha \circ \tau \circ \beta(x,-\xi)|^{2} \Phi^{\dagger} \circ \beta(x,-\xi) \cdot \Phi \circ \beta \circ \mathcal{H}^{-1}(x,-\xi) .
\end{aligned}
$$

It can be shown that with properly chosen $\alpha$, the analysis of the operator $\mathfrak{N}_{\alpha}$ becomes possible and we can recover singularities of $f$ from $\mathfrak{N}_{\alpha} f$.

We now give two definitions whose discussions are postponed to Section 6 .

Definition 3.3. A fold vector $\xi \in T_{x}^{*} \mathbb{R}^{d}$ is called fold-regular if there exists a neighborhood $U(x)$ of $x$ such that the operator germ $\mathfrak{N}_{\xi}$ is compact from $L^{2}\left(\Omega_{\epsilon_{0}}, \mathbb{R}^{2 d}\right)$ to $H^{1}\left(U(x), \mathbb{R}^{2 d}\right)$ (or from $H^{s}\left(\Omega_{\epsilon_{0}}, \mathbb{R}^{2 d}\right)$ to $H^{s+1}\left(U(x), \mathbb{R}^{2 d}\right)$ for all $s \in \mathbb{R}$ ). 
Definition 3.4. A point $x$ is called fold-regular if there exists a compact subset $\mathcal{Z}(x) \subset S_{x}^{*} \mathbb{R}^{d}$ such that the following two conditions are satisfied:

(1) For each $\xi \in \mathcal{Z}(x)$, there exist either no singular vectors or singular vectors of fold-regular type along the ray $\{t \xi: t \in \mathbb{R}\}$ for the map $\phi(x, \cdot)$;

(2) $\forall \xi \in S_{x}^{*} \mathbb{R}^{d}, \exists \theta \in \mathcal{Z}(x)$, such that $\theta \perp \xi$.

We remark that $\mathcal{Z}(x)$ parameterizes a subset of geodesics that pass through $x$ and along which there exist either no caustics or caustics of fold-regular type. A set of geodesics satisfying condition (2) in the above definition is called complete.

We now present the main result on the local stability estimate for the geodesic $\mathrm{X}$-ray transform operator. The proof is given in Section 6 .

Theorem 3.2. Let $x_{*}$ be a fold-regular point, then there exist a cut-off function $\alpha \in C_{0}^{\infty}\left(S_{-}^{*} \Gamma\right)$, a neighborhood $U\left(x_{*}\right)$ of $x_{*}$, a compact operator $\mathfrak{N}_{2, \alpha}$ from $L^{2}\left(\Omega_{\epsilon_{0}}, \mathbb{R}^{2 d}\right)$ to $H^{1}\left(U\left(x_{*}\right), \mathbb{R}^{2 d}\right)$ and a smoothing operator $\Re$ from $\mathcal{E}^{\prime}\left(\Omega, \mathbb{R}^{2 d}\right)$ into $C^{\infty}\left(\overline{U\left(x_{*}\right)}, \mathbb{R}^{2 d}\right)$, such that for any $U_{0}\left(x_{*}\right) \Subset U\left(x_{*}\right)$ the following holds:

$$
\begin{aligned}
\|f\|_{H^{s}\left(U_{0}\left(x_{*}\right), \mathbb{R}^{2 d}\right)} \lesssim & \left\|\mathfrak{N}_{\alpha} f\right\|_{H^{s+1}\left(U\left(x_{*}\right), \mathbb{R}^{2 d}\right)}+\left\|\mathfrak{N}_{2, \alpha} f\right\|_{H^{s+1}\left(U\left(x_{*}\right), \mathbb{R}^{2 d}\right)} \\
& +\|\mathfrak{R} f\|_{H^{s}\left(U\left(x_{*}\right), \mathbb{R}^{2 d}\right)}
\end{aligned}
$$

for all $f \in \mathcal{D}^{\prime}\left(\Omega_{\epsilon_{0}}, \mathbb{R}^{2 d}\right)$ and $s \in \mathbb{R}$.

\subsection{Sensitivity of recovering the velocity field from the DDtN map.}

Definition 3.5. An admissible velocity field $c$ is called fold-regular if all points in $\Omega$ are fold-regular with respect to the Hamiltonian flow $\mathcal{H}_{c}^{t}$.

We have established the following main result on the sensitivity of recovering the velocity field from the DDtN map. For simplicity we only consider the case $d=3$; similar results also hold for $d>3$. The proof is given in Section 7 .

Theorem 3.3. Let $c$ and $\tilde{c}$ be two velocity fields in the class $\mathfrak{A}\left(\epsilon_{0}, \Omega, M_{0}, T\right)$. Assume that the velocity field $c$ is smooth and is fold-regular. Then there exist a finite dimensional subspace $\mathfrak{L} \subset L^{2}\left(\Omega_{\epsilon_{0}}, \mathbb{R}^{3}\right)$, and a constant $\delta>0$ such that for all $\tilde{c}$ sufficiently close to $c$ in $H^{\frac{17}{2}}(\Omega)$ and satisfying $\nabla\left(\ln c^{2}-\ln \tilde{c}^{2}\right) \perp \mathfrak{L}$, $\left\|\Lambda_{\tilde{c}}-\Lambda_{c}\right\|_{H_{0}^{1}\left[0,3 \epsilon_{1} / 4\right] \times \Gamma \rightarrow L^{2}\left(\left[0, T+\epsilon_{1}\right] \times \Gamma\right)} \leq \delta$ implies that $c=\tilde{c}$.

\section{Gaussian beam solutions to the wave equation}

Let $c$ be a velocity field in the class $\mathfrak{A}\left(\epsilon_{0}, \Omega, M_{0}, T\right)$. We construct Gaussian beam solutions to the wave equation system (11)-(3) in this section.

We first construct a Gaussian beam in $\mathbb{R}^{d}$. Following [20, we define $G(x, \xi)=$ $|\xi|=c(x) \sqrt{\xi_{1}^{2}+\cdots+\xi_{d}^{2}}$. For a given $\left(x_{0}, \xi_{0}\right) \in S_{-}^{*} \Gamma$, let $(x(t), \xi(t), M(t), a(t))$ be the solution to the following ODE system:

$$
\begin{aligned}
\dot{x} & =G_{p}, \quad x\left(t_{0}\right)=x_{0}, \\
\dot{\xi} & =-G_{x}, \quad \xi\left(t_{0}\right)=\xi_{0}, \\
\dot{M} & =-G_{x \xi}^{\dagger} M-M G_{\xi x}-M G_{\xi \xi} M-G_{x x}, \quad M\left(t_{0}\right)=\sqrt{-1} \cdot I d, \\
\dot{a} & =-\frac{a}{2 G}\left(c^{2} \operatorname{trace}(M)-G_{x}^{\dagger} G_{\xi}-G_{\xi}^{\dagger} M G_{\xi}\right), \quad a\left(t_{0}\right)=\lambda^{\frac{d}{4}} .
\end{aligned}
$$


The corresponding Gaussian beam with frequency $\lambda(\lambda \gg 1)$ is given as follows:

$$
g(t, x, \lambda)=a(t) e^{i \lambda \tau(t, x)},
$$

where $\tau(t, x)=\xi(t) \cdot(x-x(t))+\frac{1}{2}(x-x(t))^{\dagger} M(t)(x-x(t))$.

Now, let the beam $g$ impinge on the surface $\Gamma$ transversely; we want to construct the reflected beam $g^{-}$. Let the ray $x(t)$ hit $\Gamma$ at the point $x\left(t_{1}\right)=x_{1}$. Write $\xi\left(t_{1}\right)=\xi_{1}$. We parameterize $\Gamma$ in a neighborhood of $x_{1}$, say $V\left(x_{1}\right)$, by a smooth diffeomorphism $F: U\left(x_{1}\right) \rightarrow V\left(x_{1}\right)$, where $U\left(x_{1}\right)$ is a neighborhood of the origin in $\mathbb{R}^{d-1}$. We require that $F(0)=x_{1}$. With the coordinate $x=F(y)$, we can rewrite functions when their spatial variables are restricted to the boundary $\Gamma$. For example, we rewrite

$$
g(t, x)=g(t, F(y))=\hat{g}(t, y), \quad \tau(t, x)=\tau(t, F(y))=\hat{\tau}(t, y), \quad \text { for } x \in V\left(x_{1}\right) .
$$

We remark that here and throughout this section and the next, unless specified otherwise, we use the notation $\hat{f}$ to denote the function $f$ restricted to the spacetime boundary $\Gamma \times[0, T]$ under the coordinate $x=F(y)$ for the spatial variables.

By direct calculation, we have

$$
\begin{aligned}
\hat{\tau}\left(t_{1}, y\right)= & \hat{\tau}\left(t_{1}, 0\right)+\left(\frac{\partial \hat{\tau}}{\partial(t, y)}\left(t_{1}, 0\right)\right)^{\dagger}\left(t-t_{1}, y\right)+\left(t-t_{1}, y\right) \frac{\partial^{2} \hat{\tau}}{\partial(t, y)^{2}}\left(t_{1}, 0\right)\left(t-t_{1}, y\right)^{\dagger} \\
& +O\left(\left|\left(t-t_{1}, y\right)\right|^{3}\right) \\
= & \left\langle\left(-1, \frac{\partial F}{\partial y}(0)^{\dagger} \xi_{1}\right),\left(t-t_{1}, y\right)\right\rangle+\left(t-t_{1}, y\right) \hat{M}\left(t_{1}\right)\left(t-t_{1}, y\right)^{\dagger} \\
& +O\left(\left|\left(t-t_{1}, y\right)\right|^{3}\right),
\end{aligned}
$$

where the matrix $\hat{M}\left(t_{1}\right)$ is defined as the Hessian of the phase $\hat{\tau}$ at $\left(t_{1}, 0\right)$, i.e., $\hat{M}\left(t_{1}\right)=\frac{\partial^{2} \hat{\tau}}{\partial(t, y)^{2}}\left(t_{1}, 0\right)$. It is clear that $\hat{M}\left(t_{1}\right)$ is determined by $M\left(t_{1}\right)$ and the coordinate function $x=F(y)$. Using the assumption that the ray $x(t)$ intersects $\Gamma$ transversely, we can conclude that $\Im \hat{M}\left(t_{1}\right)>0$. Moreover, if condition (8) is satisfied, we have

$$
\Im \hat{M}\left(t_{1}\right)>C
$$

for some $C>0$ depending only on $\epsilon_{0}$ and $M_{0}$.

We proceed to construct the reflected beam $g^{-}$. Write

$$
g^{-}(t, x, \lambda)=a^{-}(t) e^{i \lambda \tau^{-}(t, x)}
$$

with

$$
\tau^{-}(t, x)=\xi^{-}(t) \cdot\left(x-x^{-}(t)\right)+\frac{1}{2}\left(x-x^{-}(t)\right)^{\dagger} M^{-}(t)\left(x-x^{-}(t)\right) .
$$

We need to find $\left(x^{-}\left(t_{1}\right), \xi^{-}\left(t_{1}\right), a^{-}\left(t_{1}\right), M^{-}\left(t_{1}\right)\right)$ such that the $g^{-}+g \approx 0$ on the boundary. Following [2], we impose the following condition:

$$
\partial_{t, y}^{\alpha} \hat{\tau}\left(t_{1}, 0\right)=\partial_{t, y}^{\alpha} \hat{\tau}^{-}\left(t_{1}, 0\right), \quad \text { for all }|\alpha| \leq 2 .
$$


As a result, we obtain $\xi^{-}\left(t_{1}\right)=\xi_{1}^{-}=\xi_{1}-2\left\langle\xi_{1}, \nu\left(x_{1}\right)\right\rangle \nu\left(x_{1}\right)$ and $\hat{M}^{-}\left(t_{1}\right)=$ $\hat{M}\left(t_{1}\right)$. Here we note that $\hat{M}^{-}\left(t_{1}\right)$ is the Hessian of the phase $\hat{\tau}^{-}$for the reflected beam at the point $\left(t_{1}, 0\right)$. Consequently, $M^{-}\left(t_{1}\right)$ is also determined. Finally, set $x^{-}\left(t_{1}\right)=x_{1}$ and $a^{-}\left(t_{1}\right)=-a\left(t_{1}\right)$. Then all of the four components of $\left(x^{-}\left(t_{1}\right), \xi^{-}\left(t_{1}\right), a^{-}\left(t_{1}\right), M^{-}\left(t_{1}\right)\right)$ are constructed. Afterward, we solve an ODE system to get $\left(x^{-}(t), \xi^{-}(t), M^{-}(t), a^{-}(t)\right)$ as we did for the beam $g$. This completes the construction for the reflected beam $g^{-}$.

We now present some properties about the constructed beam. The following lemma is crucial in the subsequent estimates. We refer to 22 for the proof.

Lemma 4.1. Both the matrices $M(t)$ and $M^{-}(t)$ are uniformly bounded for $t \in$ $\left[0, T+\epsilon_{1}\right]$. Moreover, there exists $C>0$, depending on $M_{0}$ and $\epsilon_{0}$, such that $\Im M(t)>C$ and $\Im M^{-}(t)>C$ for all $t \in\left[0, T+\epsilon_{1}\right]$.

We next introduce two auxiliary beams $\hat{g}_{*}(t, y, \lambda)=a\left(t_{1}\right) e^{i \lambda \hat{\tau}_{*}}$ and $\hat{g}_{*}^{-}(t, y, \lambda)=$ $a^{-}\left(t_{1}\right) e^{i \lambda \hat{\tau}_{*}^{-}}$, where

$$
\begin{gathered}
\hat{\tau}_{*}=\left\langle\left(-1, \frac{\partial F}{\partial y}(0)^{\dagger} \xi_{1}\right),\left(t-t_{1}, y\right)\right\rangle+\left(t-t_{1}, y\right) \hat{M}\left(t_{1}\right)\left(t-t_{1}, y\right)^{\dagger}, \\
\hat{\tau}_{*}^{-}=\left\langle\left(-1, \frac{\partial F}{\partial y}(0)^{\dagger} \xi_{1}^{-}\right),\left(t-t_{1}, y\right)\right\rangle+\left(t-t_{1}, y\right) \hat{M}^{-}\left(t_{1}\right)\left(t-t_{1}, y\right)^{\dagger} .
\end{gathered}
$$

Compared to the Gaussian beams $\hat{g}$ and $\hat{g}^{-}$, the axillary beams $\hat{g}_{*}$ and $\hat{g}_{*}^{-}$have a frozen amplitude at $t=t_{1}$ and a phase function with only quadratic terms. From the proceeding construction of the reflected beam, it is clear that $\hat{\tau}_{*}=\hat{\tau}_{*}^{-}$ and $\hat{g}_{*}=-\hat{g}_{*}^{-}$. Moreover, as is shown in the next lemma, Lemma 4.2. the two axillary beams $\hat{g}_{*}$ and $\hat{g}_{*}^{-}$are good approximations to the incident beam $\hat{g}$ and reflected beam $\hat{g}^{-}$on the space-time boundary respectively. This property is used for estimating the interactions of Gaussian beams on the space-time boundary (see Step 3 in the proof of Theorem 3.1).

\section{Lemma 4.2.}

$$
\begin{aligned}
\hat{g}(t, y, \lambda) & =\hat{g}_{*}(t, y, \lambda)+O(\sqrt{\lambda}) \quad \text { in } H^{1}\left(\left(3 \epsilon_{1} / 4, t_{1}+\epsilon_{1} / 2\right) \times U\left(x_{1}\right)\right), \\
\hat{g}^{-}(t, y, \lambda) & =\hat{g}_{*}^{-}(t, y, \lambda)+O(\sqrt{\lambda}) \quad \text { in } \quad H^{1}\left(\left(3 \epsilon_{1} / 4, t_{1}+\epsilon_{1} / 2\right) \times U\left(x_{1}\right)\right) .
\end{aligned}
$$

Proof: See Appendix 8.2

Note that $\|\hat{g}(t, y, \lambda)\|_{L^{2}\left(\left(t_{1}-\epsilon_{1} / 2, t_{1}+\epsilon_{1} / 2\right) \times U\left(x_{1}\right)\right)} \approx 1$. As a direct consequence of Lemma 4.2. we obtain the following norm estimate for the beam $g$ restricted to the boundary $\Gamma$.

\section{Lemma 4.3.}

$$
\|g(\cdot, \cdot, \lambda)\|_{L^{2}\left(\left(t_{1}-\epsilon_{1} / 2, t_{1}+\epsilon_{1} / 2\right) \times V\left(x_{1}\right)\right)} \approx 1 .
$$


We now present an $H^{1}$-norm estimate for $g^{-}+g$ and an approximation for the Neumann data $\frac{\partial g}{\partial \nu}^{-}+\frac{\partial g}{\partial \nu}$ on the boundary.

\section{Lemma 4.4 .}

$$
\begin{aligned}
g^{-}(t, x, \lambda)+g(t, x, \lambda)= & O(\sqrt{\lambda}) \quad \text { in } H^{1}\left(\left(3 \epsilon_{1} / 4, t_{1}+\epsilon_{1} / 2\right) \times V\left(x_{1}\right)\right) ; \\
\frac{\frac{\partial g}{\partial \nu}^{-}+\frac{\partial g}{\partial \nu}=}{\partial \nu} & 2 i \lambda \cdot\left\langle\xi_{1}, \nu\left(x_{1}\right)\right\rangle \\
& +O(\sqrt{\lambda}) \text { in } L^{2}\left(\left(3 \epsilon_{1} / 4, t_{1}+\epsilon_{1} / 2\right) \times V\left(x_{1}\right)\right) .
\end{aligned}
$$

\section{Proof: See Appendix 8.2}

Now, we are ready to construct Gaussian beam solutions to the initial boundary value problem of the wave system (11)-(3). We first choose $\chi_{\epsilon_{1}}(t) \in C_{0}^{\infty}(\mathbb{R})$ such that $\chi_{\epsilon_{1}}(t)=1$ for $t \in\left(\epsilon_{1} / 4, \epsilon_{1} / 2\right)$ and $\chi_{\epsilon_{1}}(t)=0$ for $t \in(-\infty, 0) \bigcup\left(3 \epsilon_{1} / 4, \infty\right)$. Let $\left(x_{0}, \xi_{0}\right) \in S_{-}^{*} \Gamma$ and $\left(x_{0}^{*}, \xi_{0}^{*}\right)=\mathcal{H}^{-\frac{\epsilon_{1}}{4}}\left(x_{0}, \xi_{0}\right)=\left(x_{0}-\frac{\epsilon_{1} \cdot \xi_{1}}{4}, \xi_{0}\right)$. Let $g$ be the Gaussian beam constructed with the initial data $x(0)=x_{0}^{*}, \xi(0)=\xi_{0}^{*}, M(0)=$ $i \cdot I d$ and $a(0)=\lambda^{\frac{d}{4}}$. The beam $g$ is reflected by $\Gamma$ at $\left(x_{1}, \xi_{1}\right)=\mathfrak{S}_{c}\left(x_{0}, \xi_{0}\right)=$ $\mathcal{H}_{c}^{l\left(x_{0}, \xi_{0}\right)}\left(x_{0}, \xi_{0}\right)$ at $t_{1}=l\left(x_{0}, \xi_{0}\right)+\frac{\epsilon_{1}}{4}$. We construct the reflected beam $g^{-}$by the preceding procedure. Let $u$ be the exact solution to the wave system (11)-(3) with

$$
f(t, x, \lambda)=g(t, x, \lambda) \cdot \chi_{\epsilon_{1}}(t) .
$$

Then $u=g+g^{-}+R$, where the remaining term $R$ satisfies the following equation system:

$$
\begin{aligned}
\mathcal{P} R & =-\mathcal{P}\left(g+g^{-}\right), \quad(t, x) \in \Omega \times\left(0, t_{1}+\epsilon_{1} / 2\right), \\
R(0, x, \lambda) & =-\left(g+g^{-}\right)(0, x, \lambda), \quad x \in \Omega, \\
R_{t}(0, x, \lambda) & =-\left(g_{t}+g_{t}^{-}\right)(0, x, \lambda), \quad x \in \Omega, \\
R(t, x, \lambda) & =-g(t, x, \lambda)\left(1-\chi_{\epsilon_{1}}(t)\right)-g^{-}(t, x, \lambda), \quad(t, x) \in\left(0, t_{1}+\epsilon_{1} / 2\right) \times \Gamma .
\end{aligned}
$$

Here $\mathcal{P}$ stands for the wave operator $\frac{1}{c^{2}(x)} \partial_{t t}-\Delta$.

\section{Lemma 4.5.}

$$
\left\|\frac{\partial R}{\partial \nu}\right\|_{L^{2}\left(\left[0, t_{1}+\epsilon_{1} / 2\right] \times \Gamma\right)} \leq C \sqrt{\lambda}
$$

for some constant $C>0$ depending on $\epsilon_{0}$ and $M_{0}$.

Proof. We apply Theorem 4.1 in [16] to derive the estimate. Note that the compatibility condition is satisfied on the boundary at time $t=0$. It remains to show that the following four estimates hold:

$$
\begin{aligned}
\left\|\mathcal{P}\left(g+g^{-}\right)\right\|_{C\left(\left[0, t_{1}+\epsilon_{1} / 2\right] ; L^{2}(\Omega)\right)} & \lesssim \sqrt{\lambda}, \\
\left\|\left(g+g^{-}\right)(0, \cdot, \lambda)\right\|_{H^{1}(\Omega)} & \lesssim \sqrt{\lambda}, \\
\left\|\left(g_{t}+g_{t}^{-}\right)(0, \cdot, \lambda)\right\|_{L^{2}(\Omega)} & \lesssim \sqrt{\lambda}, \\
\left\|g(t, x, \lambda)\left(1-\chi_{\epsilon_{1}}(t)\right)-g^{-}(t, x, \lambda)\right\|_{H^{1}\left(\left[0, t_{1}+\epsilon_{1} / 2\right] \times \Gamma\right)} & \lesssim \sqrt{\lambda} .
\end{aligned}
$$


First, (35) follows from the standard estimate for Gaussian beams; see, for example, [5]. We next show (36). By Lemma 4.1, there exists a constant $C>0$ depending on $M_{0}$ and $\epsilon_{0}$ such that the following two inequalities hold:

$$
\begin{aligned}
|g(t, x, \lambda)| & \lesssim \lambda^{\frac{d}{4}} \cdot e^{-C \lambda \cdot\left|x-x^{-}(t)\right|^{2}}, \\
\left|g^{-}(t, x, \lambda)\right| & \lesssim \lambda^{\frac{d}{4}} \cdot e^{-C \lambda \cdot\left|x-x^{-}(t)\right|^{2}} .
\end{aligned}
$$

Thus the beam $g$ and $g^{-}$are exponentially decaying away from the ray $x(t)$ and $x^{-}(t)$, respectively. Using this property, it is straightforward to show that $\|g(0, \cdot, \lambda)\|_{H^{1}(\Omega)} \lesssim 1$ and $\left\|g^{-}(0, \cdot, \lambda)\right\|_{H^{1}(\Omega)} \lesssim 1$, whence (36) and (37) follows.

Now, we show (38). We divide the domain $\left(0, t_{1}+\epsilon_{1} / 2\right) \times \Gamma$ into three parts:

$$
\Sigma_{1}=\left(0, \epsilon_{1} / 2\right) \times \Gamma, \quad \Sigma_{2}=\left(\epsilon_{1} / 2, t_{1}-\epsilon_{1} / 2\right) \times \Gamma, \quad \Sigma_{3}=\left(t_{1}-\epsilon_{1} / 2, t_{1}+\epsilon_{1} / 2\right) \times \Gamma .
$$

We show that inequality (38) holds on each part.

For $(t, x) \in \Sigma_{1}$, we have $1-\chi_{\epsilon_{1}}(t)=0$. Consequently,

$$
g(t, x)\left(1-\chi_{\epsilon_{1}}(t)\right)-g^{-}(t, x, \lambda)=g^{-}(t, x, \lambda) .
$$

By the exponential decaying property of $g^{-}$, we obtain that

$$
\left\|g(t, x)\left(1-\chi_{\epsilon_{1}}(t)\right)-g^{-}(t, x, \lambda)\right\|_{H^{1}\left(\Sigma_{1}\right)} \lesssim \sqrt{1} .
$$

For $(t, x) \in \Sigma_{2}$, by the exponential decaying property for both $g$ and $g^{-}$again, we obtain

$$
\left\|g(t, x, \lambda)\left(1-\chi_{\epsilon_{1}}(t)\right)-g^{-}(t, x, \lambda)\right\|_{H^{1}\left(\Sigma_{2}\right)} \lesssim 1 .
$$

Finally, for $(t, x) \in \Sigma_{3}$, note that $t_{1}-\frac{\epsilon_{1}}{2}=l\left(x_{0}, \xi_{0}\right)+\frac{\epsilon_{1}}{4}-\frac{\epsilon_{1}}{2} \geq \frac{3 \epsilon_{1}}{4}$. We can apply Lemma 4.4 to the part $x \in V\left(x_{1}\right)$ and the exponential decaying property for both $g$ and $g^{-}$to the remaining part to conclude that

$$
\left\|g(t, x, \lambda)\left(1-\chi_{\epsilon_{1}}(t)\right)-g^{-}(t, x, \lambda)\right\|_{H^{1}\left(\Sigma_{3}\right)} \lesssim \sqrt{\lambda} .
$$

This completes the proof of (38) and hence the lemma.

\section{Proof of Theorem 3.1}

Proof of Theorem 3.1. For any $\left(x_{0}, \xi_{0}\right) \in S_{-}^{*} \Gamma$, let $\left(x_{1}, \xi_{1}\right)=\mathfrak{S}_{c}\left(x_{0}, \xi_{0}\right)=$ $\mathcal{H}_{c}^{l\left(x_{0}, \xi_{0}\right)}\left(x_{0}, \xi_{0}\right)$ and $\left(\tilde{x}_{1}, \tilde{\xi}_{1}\right)=\mathfrak{S}_{\tilde{c}}\left(x_{0}, \xi_{0}\right)=\mathcal{H}_{\tilde{c}}^{\tilde{l}\left(x_{0}, \xi_{0}\right)}\left(x_{0}, \xi_{0}\right)$. We need to show that $\left(l\left(x_{0}, \xi_{0}\right), x_{1}, \xi_{1}\right)=\left(\tilde{l}\left(x_{0}, \xi_{0}\right), \tilde{x}_{1}, \tilde{\xi}_{1}\right)$ if $\left\|\Lambda_{\tilde{c}}-\Lambda_{c}\right\|$ is sufficiently small. We do this in the following steps.

Step 1. Let $t_{1}=l\left(x_{0}, \xi_{0}\right)+\frac{\epsilon_{1}}{4}$ and $\tilde{t}_{1}=\tilde{l}\left(x_{0}, \xi_{0}\right)+\frac{\epsilon_{1}}{4}$. Without loss of generality, we may assume that $t_{1} \leq \tilde{t}_{1}$. Let $V\left(x_{1}\right)$ be a neighborhood of $x_{1}$ in $\Gamma$ which is parameterized by a smooth function $F: U\left(x_{1}\right) \rightarrow V\left(x_{1}\right)$ as before. Note that if $\tilde{x}_{1}$ does not belong to $V\left(x_{1}\right)$, then we can show that $\left\|\Lambda_{\tilde{c}}-\Lambda_{c}\right\|$ is bounded from below by some positive constant and hence the result of Theorem 3.1 is obvious. Therefore we may assume that $\tilde{x}_{1} \in V\left(x_{1}\right)$. Let $\tilde{x}_{1}=F(\delta y)$. We construct the initial beam $g$, the reflected beam $g^{-}$, the boundary Dirichlet data $f$, the solution $u$ to the wave equation with velocity field $c$ and remaining term $R$ as in the previous section. We similarly construct $\tilde{g}, \tilde{g}^{-}, \tilde{u}$ and $\tilde{R}$ to the system with velocity field $\tilde{c}$ and with boundary Dirichlet data $\tilde{f}=f$. 
Step 2. Denote by $I\left(t_{1}, \epsilon_{1} / 2\right)$ the interval $\left(t_{1}-\epsilon_{1} / 2, t_{1}+\epsilon_{1} / 2\right)$. Since $t_{1} \leq \tilde{t}_{1}$ and $l\left(x_{0}, \xi_{0}\right) \geq \epsilon_{1}$, we have $I\left(t_{1}, \epsilon_{1} / 2\right) \subset\left(3 \epsilon_{1} / 4, t_{1}+\epsilon_{1} / 2\right)$ and $I\left(t_{1}, \epsilon_{1} / 2\right) \subset\left(3 \epsilon_{1} / 4, \tilde{t}_{1}+\right.$ $\left.\epsilon_{1} / 2\right)$. Then we can apply (34) and Lemma 4.5 to obtain

$$
\begin{aligned}
\left(\Lambda_{\tilde{c}}-\Lambda_{c}\right) f & =\frac{\partial u}{\partial \nu}-\frac{\partial \tilde{u}}{\partial \nu} \\
& =\frac{\partial\left(g+g^{-}\right)}{\partial \nu}-\frac{\partial\left(\tilde{g}+\tilde{g}^{-}\right)}{\partial \nu}+\frac{\partial R}{\partial \nu}-\frac{\partial \tilde{R}}{\partial \nu} \\
& =2 i \lambda \cdot\left\{\left\langle\xi_{1}, \nu\left(x_{1}\right)\right\rangle \cdot g-\left\langle\tilde{\xi}_{1}, \nu\left(\tilde{x}_{1}\right)\right\rangle \cdot \tilde{g}\right\}+O(\sqrt{\lambda})
\end{aligned}
$$

in $L^{2}\left(I\left(t_{1}, \epsilon_{1} / 2\right) \times V\left(x_{1}\right)\right)$.

It follows that

$$
\begin{aligned}
\left\langle\left(\Lambda_{\tilde{c}}-\Lambda_{c}\right) f, g\right\rangle_{L^{2}\left(I\left(t_{1}, \epsilon_{1} / 2\right) \times V\left(x_{1}\right)\right)}= & 2 i \lambda \cdot\left[\left\langle\xi_{1}, \nu\left(x_{1}\right)\right\rangle \cdot\langle g, g\rangle_{L^{2}\left(I\left(t_{1}, \epsilon_{1} / 2\right) \times V\left(x_{1}\right)\right)}\right. \\
& \left.-\left\langle\tilde{\xi}_{1}, \nu\left(\tilde{x}_{1}\right)\right\rangle \cdot\langle\tilde{g}, g\rangle_{L^{2}\left(I\left(t_{1}, \epsilon_{1} / 2\right) \times V\left(x_{1}\right)\right)}\right] \\
& +O(\sqrt{\lambda}) .
\end{aligned}
$$

Note that

$$
\begin{aligned}
\mid\left\langle\left(\Lambda_{\tilde{c}}-\Lambda_{c}\right) f, g\right\rangle_{L^{2}\left(I\left(t_{1}, \epsilon_{1} / 2\right) \times V\left(x_{1}\right)\right)} \leq & \left\|\left(\Lambda_{\tilde{c}}-\Lambda_{c}\right) f\right\|_{L^{2}\left(I\left(t_{1}, \epsilon_{1} / 2\right) \times V\left(x_{1}\right)\right)} \\
& \cdot\|g\|_{L^{2}\left(I\left(t_{1}, \epsilon_{1} / 2\right) \times V\left(x_{1}\right)\right)} \\
\leq & \left\|\left(\Lambda_{\tilde{c}}-\Lambda_{c}\right) f\right\|_{L^{2}\left(\left(0, T+\epsilon_{1}\right) \times \Gamma\right)} \\
& \cdot\|g\|_{L^{2}\left(I\left(t_{1}, \epsilon_{1} / 2\right) \times V\left(x_{1}\right)\right)} \\
\leq & \left\|\Lambda_{\tilde{c}}-\Lambda_{c}\right\|_{H_{0}^{1}\left(\left[0,3 \epsilon_{1} / 4\right] \times \Gamma\right) \rightarrow L^{2}\left(\left[0, T+\epsilon_{1}\right] \times \Gamma\right)} \\
& \cdot\|f\|_{H_{0}^{1}\left(\left[0,3 \epsilon_{1} / 4\right] \times \Gamma\right)} \cdot\|g\|_{L^{2}\left(I\left(t_{1}, \epsilon_{1} / 2\right) \times V\left(x_{1}\right)\right)} \\
\lesssim & \lambda \cdot\left\|\Lambda_{\tilde{c}}-\Lambda_{c}\right\|_{H_{0}^{1}\left(\left[0,3 \epsilon_{1} / 4\right] \times \Gamma\right) \rightarrow L^{2}\left(\left[0, T+\epsilon_{1}\right] \times \Gamma\right)} .
\end{aligned}
$$

Thus the following inequality holds:

$$
\begin{aligned}
& \left|\left\langle\xi_{1}, \nu\left(x_{1}\right)\right\rangle \cdot\langle g, g\rangle_{L^{2}\left(I\left(t_{1}, \epsilon_{1} / 2\right) \times V\left(x_{1}\right)\right)}\right|-\left|\left\langle\tilde{\xi}_{1}, \nu\left(\tilde{x}_{1}\right)\right\rangle \cdot\langle\tilde{g}, g\rangle_{L^{2}\left(I\left(t_{1}, \epsilon_{1} / 2\right) \times V\left(x_{1}\right)\right)}\right| \\
& \quad \leq \frac{C}{\sqrt{\lambda}}+C \cdot\left\|\Lambda_{\tilde{c}}-\Lambda_{c}\right\|_{H_{0}^{1}\left(\left[0,3 \epsilon_{1} / 4\right] \times \Gamma\right) \rightarrow L^{2}\left(\left[0, T+\epsilon_{1}\right] \times \Gamma\right)}
\end{aligned}
$$

for some constant $C>0$.

Step 3. We now estimate the two terms on the left hand side of the inequality (39). First, by (8) and Lemma 4.3, we have

$$
\left|\left\langle\xi_{1}, \nu\left(x_{1}\right)\right\rangle \cdot\right|\langle g, g\rangle_{L^{2}\left(I\left(t_{1}, \epsilon_{1} / 2\right) \times V\left(x_{1}\right)\right)} \mid \approx 1 .
$$

We next estimate $\langle\tilde{g}, g\rangle_{L^{2}\left(I\left(t_{1}, \epsilon_{1} / 2\right) \times V\left(x_{1}\right)\right)}$. In the coordinate $x=F(y)$, by Lemma 4.2, we have

$$
\langle\hat{\tilde{g}}, \hat{g}\rangle_{L^{2}\left(I\left(t_{1}, \epsilon_{1} / 2\right) \times U\left(x_{1}\right)\right)}=\left\langle\hat{\tilde{g}}_{*}, \hat{g}_{*}\right\rangle_{L^{2}\left(I\left(t_{1}, \epsilon_{1} / 2\right) \times U\left(x_{1}\right)\right)}+O\left(\frac{1}{\sqrt{\lambda}}\right),
$$


where $\hat{g}_{*}$ and $\hat{\tilde{g}}_{*}$ are the auxiliary beams associated with the beams $\hat{g}$ and $\hat{\tilde{g}}$, respectively. We write $\hat{g}_{*}=a\left(t_{1}\right) e^{i \lambda \hat{\tau}_{*}}$ and $\hat{\tilde{g}}_{*}=\tilde{a}\left(t_{1}\right) e^{i \lambda \hat{\tilde{\tau}}_{*}}$ with

$$
\begin{aligned}
& \hat{\tau}_{*}=\left\langle\left(-1, \frac{\partial F}{\partial y}(0)^{\dagger} \xi_{1}\right),\left(t-t_{1}, y\right)\right\rangle+\left(t-t_{1}, y\right) \hat{M}\left(t_{1}\right)\left(t-t_{1}, y\right)^{\dagger} \\
& \hat{\tilde{\tau}}_{*}=\left\langle\left(-1, \frac{\partial F}{\partial y}(\delta y)^{\dagger} \tilde{\xi}_{1}\right),\left(t-\tilde{t}_{1}, y-\delta y\right)\right\rangle+\left(t-\tilde{t}_{1}, y-\delta y\right) \hat{\tilde{M}}\left(t_{1}\right)\left(t-\tilde{t}_{1}, y-\delta y\right)^{\dagger} .
\end{aligned}
$$

By Lemma 8.1 in Appendix 8.3 , we have

$$
\left|\left\langle\hat{\tilde{g}}_{*}, \hat{g}_{*}\right\rangle_{L^{2}\left(I\left(t_{1}, \epsilon_{1} / 2\right) \times U\left(x_{1}\right)\right)}\right| \lesssim e^{-c_{0} \lambda|\delta z|},
$$

where $c_{0}$ is a positive constant depending only on $\|c\|_{C^{3}}+\|\tilde{c}\|_{C^{3}}$ and $|\delta z|=$ $\left|t_{1}-\tilde{t}_{1}\right|^{2}+|\delta y|^{2}+\left|\frac{\partial F}{\partial y}(\delta y)^{\dagger} \tilde{\xi}_{1}-\frac{\partial F}{\partial y}(0)^{\dagger} \xi_{1}\right|^{2}$. It follows from (41) and (42) that

$$
\left|\langle\tilde{g}, g\rangle_{L^{2}\left(I\left(t_{1}, \epsilon_{1} / 2\right) \times V\left(x_{1}\right)\right)}\right| \lesssim e^{-c_{0} \lambda|\delta z|}+O\left(\frac{1}{\sqrt{\lambda}}\right) .
$$

Step 4. Combining (39), (40) and (43), we see that

$$
e^{-c_{0} \lambda|\delta z|} \gtrsim C_{1}-C_{2}\left\|\Lambda_{\tilde{c}}-\Lambda_{c}\right\|_{H_{0}^{1}\left(\left[0,3 \epsilon_{1} / 4\right] \times \Gamma\right) \rightarrow L^{2}\left(\left[0, T+\epsilon_{1}\right] \times \Gamma\right)}-C_{3} \frac{1}{\sqrt{\lambda}}
$$

for some positive constants $C_{1}, C_{2}$ and $C_{3}$ which are independent of $\left(x_{0}, \xi_{0}\right)$. By letting $\lambda \rightarrow \infty$, we conclude that $\delta z=0$ if

$$
\left\|\Lambda_{\tilde{c}}-\Lambda_{c}\right\|_{H_{0}^{1}\left(\left[0,3 \epsilon_{1} / 4\right] \times \Gamma\right) \rightarrow L^{2}\left(\left[0, T+\epsilon_{1}\right] \times \Gamma\right)}<\frac{C_{1}}{C_{2}} .
$$

Set $\delta=\frac{C_{1}}{C_{2}}$. From $\delta z=0$ it follows that $t_{1}=\tilde{t}_{1}, \delta y=0$, and $\frac{\partial F}{\partial y}(0)^{\dagger} \tilde{\xi}_{1}-\frac{\partial F}{\partial y}(0)^{\dagger} \xi_{1}=$ 0 . It remains to show that $\tilde{\xi}_{1}=\xi_{1}$. Indeed, $\frac{\partial F}{\partial y}(0)^{\dagger} \tilde{\xi}_{1}-\frac{\partial F}{\partial y}(0)^{\dagger} \xi_{1}=0$ implies that the tangential component of $\tilde{\xi}_{1}$ and $\xi_{1}$ are equal. Besides, $\left|\xi_{1}\right|=\left|\tilde{\xi}_{1}\right|$. These together with (8) yield that $\tilde{\xi}_{1}=\xi_{1}$. This completes the proof of the theorem.

\section{Geodesic X-Ray transform with Caustics}

6.1. Local properties of the normal operator $\mathfrak{N}$. In this subsection, we present some results about the local properties of the normal operator $\mathfrak{N}$ (see (20)).

From now on, we fix $x_{*} \in \Omega$. We first decompose $\mathfrak{N}$ locally into two parts based on the separation of singularities of its Schwartz kernel. Note that the map $\phi\left(x_{*}, \cdot\right): \mathbb{R}^{d} \rightarrow \mathbb{R}^{d}$ is a diffeomorphism in a neighborhood of the origin. In fact, we can check that $\frac{\partial \phi\left(x_{*}, \cdot\right)}{\partial \xi}(0)=c\left(x_{*}\right) \cdot I d$. Similar to the proof of existence of the uniformly normal neighborhood in the Riemannian manifold [17, we can find $\epsilon_{2}>0$ and a neighborhood of $x_{*}$, say $\tilde{U}\left(x_{*}\right) \subset \mathbb{R}^{d}$, such that

$$
\left.\phi(x, \cdot)\right|_{|\xi|<2 \epsilon_{2}} \text { is a diffeomorphism for any } x \in \tilde{U}\left(x_{*}\right) .
$$

Let $\chi_{\epsilon_{2}} \in C_{0}^{\infty}(\mathbb{R})$ be such that $\chi(t)=1$ for $|t|<\epsilon_{2}$ and $\chi(t)=0$ for $|t|>2 \epsilon_{2}$. We then define

$$
\begin{aligned}
& \mathfrak{N}_{1} f(x)=\int_{T_{x}^{*} \Omega} W(x, \xi) f(\phi(x, \xi)) \chi_{\epsilon_{2}}(|\xi|) d \sigma_{x}(\xi), \\
& \mathfrak{N}_{2} f(x)=\int_{T_{x}^{*} \Omega} W(x, \xi) f(\phi(x, \xi))\left(1-\chi_{\epsilon_{2}}(|\xi|)\right) d \sigma_{x}(\xi) .
\end{aligned}
$$


Note that for any $f$ supported in $\Omega, f(\phi(x, \xi))=0$ for all $|\xi|>T$. Thus we have

$$
\mathfrak{N}_{2} f(x)=\int_{\xi \in T_{x}^{*} \Omega, \epsilon_{2}<|\xi|<T} W(x, \xi) f(\phi(x, \xi))\left(1-\chi_{\epsilon_{2}}(|\xi|)\right) d \sigma_{x}(\xi) .
$$

It is clear that $\mathfrak{N} f=\mathfrak{N}_{1} f+\mathfrak{N}_{2} f$. This gives the promised decomposition of $\mathfrak{N}$. We next study $\mathfrak{N}_{1}$ and $\mathfrak{N}_{2}$ separately.

Lemma 6.1. $\mathfrak{N}_{1}$ is an elliptic $\Psi D O$ of order -1 from $C_{0}^{\infty}\left(\tilde{U}\left(x_{*}\right), \mathbb{R}^{2 d}\right)$ to $\mathcal{D}^{\prime}\left(\tilde{U}\left(x_{*}\right), \mathbb{R}^{2 d}\right)$ with principle symbol

$$
\sigma_{p}\left(\mathfrak{N}_{1}\right)(x, \xi)=2 \pi \cdot \int_{S_{x}^{*} \Omega} \delta(\langle\xi, \theta\rangle) \Phi^{\dagger}(x, \theta) \cdot \Phi(x, \theta) d \sigma_{x}(\theta) .
$$

Proof. See [30] or [35].

We now proceed to study the operator $\mathfrak{N}_{2}$ whose property is determined by the Lagrangian map $\phi\left(x_{*}, \cdot\right)$. We shall study the operator germ $\mathfrak{N}_{2, \xi_{*}}$ for each $\xi \in T_{x_{*}}^{*} \mathbb{R}^{d}$. We first consider the case when $\xi_{*}$ is not a caustic vector; i.e., $\xi_{*}$ is a regular vector.

Lemma 6.2. Let $\xi_{*} \in S_{x_{*}}^{*} \mathbb{R}^{d}$ be a regular vector, then there exists a neighborhood $U\left(x_{*}\right)$ of $x_{*}$ and a neighborhood $B\left(x_{*}, \xi_{*}\right)$ of $\left(x_{*}, \xi_{*}\right)$ such that for any $\chi \in C_{0}^{\infty}\left(B\left(x_{*}, \xi_{*}\right)\right)$ the following operator:

$$
\mathfrak{N}_{2, \xi_{*}} f(x)=\int_{T_{x}^{*} \Omega} W(x, \xi) f(\phi(x, \xi))\left(1-\chi_{\epsilon_{2}}(|\xi|)\right) \cdot \chi(x, \xi) d \sigma_{x}(\xi)
$$

is a smoothing operator from $\mathcal{E}^{\prime}\left(\Omega, \mathbb{R}^{2 d}\right)$ into $C^{\infty}\left(\overline{U\left(x_{*}\right)}, \mathbb{R}^{2 d}\right)$.

Proof. Since $\xi_{*} \in S_{x_{*}}^{*} \mathbb{R}^{d}$ is regular, there exist a neighborhood $V\left(x_{*}\right)$ of $x_{*}$ in $\mathbb{R}^{d}$ and a neighborhood $B\left(x_{*}, \xi_{*}\right)$ of $\left(x_{*}, \xi_{*}\right)$ in $\mathbb{R}^{2 d}$ of the form $B\left(x_{*}, \xi_{*}\right)=$ $V\left(x_{*}\right) \times B_{0}\left(\xi_{*}\right)$ for some open set $B_{0}\left(\xi_{*}\right)$ in $\mathbb{R}^{d}$ such that the map $\phi(x, \cdot)$ is a diffeomorphism between $B_{0}\left(\xi_{*}\right)$ and its image for all $x \in V\left(x_{*}\right)$. We denote the inverse of the map $\phi(x, \cdot)$ by $\phi^{-1}(x, \cdot)$. By a change of coordinate $\xi=\phi^{-1}(x, y)$ and the use of some cut-off function, we can write $\mathfrak{N}_{2, \xi_{*}}$ in the following form:

$$
\mathfrak{N}_{2, \xi_{*}} f(x)=\int_{\Omega} K(x, y) f(y) d y, f \in \mathcal{E}^{\prime}\left(\Omega, \mathbb{R}^{2 d}\right)
$$

for some smooth function $K$ in $\Omega \times \Omega$. The Lemma follows immediately.

We next consider the case when $\xi_{*}$ is a fold vector. We have the following slightly modified result from 35.

Lemma 6.3. Let $\xi_{*}$ be a fold vector of the map $\phi\left(x_{*}, \cdot\right)$, then there exists a small neighborhood $U\left(x_{*}\right)$ of $x_{*}$ and a small neighborhood $B\left(x_{*}, \xi_{*}\right)$ of $\left(x_{*}, \xi_{*}\right)$ in $\mathbb{R}^{2 d}$ such that for any $\chi \in C_{0}^{\infty}\left(B\left(x_{*}, \xi_{*}\right)\right)$, the operator $\mathfrak{N}_{2, \xi_{*}}: \mathcal{E}^{\prime}\left(\Omega, \mathbb{R}^{2 d}\right) \rightarrow \mathcal{D}^{\prime}\left(U\left(x_{*}\right), \mathbb{R}^{2 d}\right)$ defined by

$\mathfrak{N}_{2, \xi_{*}} f(x)=\int_{T_{x}^{*} \Omega} W(x, \xi) f(\phi(x, \xi))\left(1-\chi_{\epsilon_{2}}(|\xi|)\right) \cdot \chi(x, \xi) d \sigma_{x}(\xi), \quad f \in \mathcal{E}^{\prime}\left(\Omega, \mathbb{R}^{2 d}\right)$, 
is an FIO of order $-\frac{d}{2}$ whose associated canonical relation is compactly supported in the following set:

$$
\begin{gathered}
\left\{(x, \xi, y, \eta) ; x \in U\left(x_{*}\right), y=\phi(x, \omega),(x, \omega) \in B\left(x_{*}, \xi_{*}\right), \operatorname{det} d_{\omega} \phi(x, \omega)=0,\right. \\
\left.\xi=-\eta_{i} \frac{\partial \phi^{i}(x, \omega)}{\partial x}, \eta \in \operatorname{Coker}\left(d_{\omega} \phi(x, \omega)\right) .\right\}
\end{gathered}
$$

6.2. Singularities of the map $\phi(x, \cdot)$. In this subsection, we present some properties about the map $\phi(x, \cdot)$ which is equivalent to the exponential map in the Riemannian manifold.

By the classification result for Lagrangian maps (see 3] and [4] for details), there is only a finite number of stable and simple singular Lagrangian map germs in dimensions between three and five and they are generic. In three dimensions, there are four types: fold, cusp, swallow-tail and D4. The others are unstable and can be removed by using arbitrarily small perturbations. We define

$\mathcal{K}(x)=\left\{\xi \in T_{x}^{*} \mathbb{R}^{d}:\right.$ the map germ $\phi(x, \cdot)$ at $\xi$ is singular $\} ;$

$\mathcal{K}_{1}(x)=\left\{\xi \in T_{x}^{*} \mathbb{R}^{d}\right.$ : the map germ $\phi(x, \cdot)$ at $\xi$ has singularity of fold type $\} ;$

$\mathcal{K}_{2}(x)=\left\{\xi \in T_{x}^{*} \mathbb{R}^{d}\right.$ : the map germ $\phi(x, \cdot)$ at $\xi$ has singularity of cusp type $\} ;$

$\mathcal{K}_{3}(x)=\left\{\xi \in T_{x}^{*} \mathbb{R}^{d}\right.$ : the map germ $\phi(x, \cdot)$ at $\xi$ has simple and stable singularities of types other than fold and cusp\}.

We say that the map $\phi(x, \cdot)$ is in a general position (or generic) if the map germ $\phi(x, \cdot)$ is simple and stable at all caustic vectors in $\mathcal{K}(x)$. By the classification result of Lagrangian maps, see for instance [3], the following result holds for the set $\mathcal{K}(x)$.

Propsition 1. Assume that the map $\phi(x, \cdot)$ is in a general position, then the sets $\mathcal{K}_{1}(x)$ and $\mathcal{K}_{2}(x)$ are smooth manifolds of dimensions $d-1$ and $d-2$, respectively. The set $\mathcal{K}_{3}(x)$ is a union of smooth manifolds of dimensions not greater than $d-3$. Especially, for $d=3$, the sets $\mathcal{K}_{1}(x), \mathcal{K}_{2}(x)$ and $\mathcal{K}_{3}(x)$ consist of smooth surfaces, smooth curves and isolated points, respectively.

In the case when the map $\phi(x, \cdot)$ is not in a general position, it is known that $\bigcup_{j=1}^{3} \mathcal{K}_{j}(x)$ is open and dense in $\mathcal{K}(x)$.

Recall that the map $\beta$ is defined by (19). Denote $\mathcal{S}_{j}(x)=\beta\left(\mathcal{K}_{j}(x)\right)$ for $j=1,2,3$. We conclude that the following result holds.

Lemma 6.4. Assume that the map $\phi(x, \cdot)$ is in the general position, then the sets $\mathcal{S}_{1}(x), \mathcal{S}_{2}(x)$ and $\mathcal{S}_{3}(x)$ are of finite $d-1, d-2$ and $d-3$ dimensional Hausdorff measures, respectively. Especially, for $d=3$, the set $\mathcal{S}_{2}(x)$ is a curve (not necessarily smooth) of finite length in $S_{x}^{*} \mathbb{R}^{3}$ and $\mathcal{S}_{3}(x)$ consists of a finite number of points.

It is clear that $\mathcal{S}_{1}(x)$ represents the set of geodesics passing through $x$ with fold caustics (and possibly other types of caustics as well). Observe that for each fold vector $\xi \in T_{x}^{*} \mathbb{R}^{d}$, there exists a neighborhood of $\xi$ in $\mathcal{K}_{1}(x)$, say $V(\xi)$, such that $V(\xi)$ is a smooth hyperplane of dimension $d-1$ in $T_{x}^{*} \mathbb{R}^{d}$ and is transversal to the ray $\{t \xi: t>0\}$. From this, we can conclude that the following result holds.

Lemma 6.5. The set $\mathcal{S}_{1}(x)$ is open in $S_{x}^{*} \mathbb{R}^{d}$. 
6.3. Discussions on the concept of fold-regular. In this subsection, we discuss the concept "fold-regular" defined in Subsection 3.3. We show that the fold-regular point is the natural object to study for geodesic X-ray transforms with caustics. We also derive some natural conditions for a point to be fold-regular. We remark that one can define "fold-regular metric" in a similar way as for the "fold-regular velocity field" in Subsection 3.4. Then "fold-regular metric" generalizes "regular metric" introduced in [29].

We take the case $d=3$, for example, similar discussions also hold for the case $d>3$. Note that the set of geodesics passing through $x$ can be parameterized by the sphere $S_{x}^{*} \mathbb{R}^{3}$ and those with fold caustics by $\mathcal{S}_{1}(x)$, which is a subset with the same dimension. In comparison, a complete set of geodesics passing through $x$ has a dimension of at least one. By Lemma 6.5, we see that in the generic case for a non-simple velocity field, the set $\mathcal{S}_{1}(x)$ is open in the sphere $S_{x}^{*} \mathbb{R}^{3}$. Thus, in order to be able to select a complete set from $S_{x}^{*} \mathbb{R}^{3}$, it is necessary to use geodesics with fold caustics in the case when there is no complete subset in the set of geodesics without caustics. However, we should also point out that there is not much need to use geodesics with other types of caustics since they correspond to a one dimensional subset on $S_{x}^{*} \mathbb{R}^{3}$. In the extreme case, when the set $\mathcal{S}_{1}(x)$ contains no complete subset, then there exists $\theta \in S_{x}^{*} \mathbb{R}^{3}$ such that all the geodesics represented by the set $\theta^{\perp}=\left\{\xi \in S_{x}^{*} \mathbb{R}^{d}, \xi \perp \theta\right\}$ have caustics other than the fold type. It is only in such an extreme case that we need to use information from geodesics with cusp caustics and hence an investigation of properties of the operator germ $\mathfrak{N}_{\xi}$ for cusp vectors becomes necessary.

We now consider the problem when a fold vector is fold-regular. In dimension $d \geq 3$, a sufficient condition for a fold vector $\xi_{*}$ to be fold-regular is the following:

$$
\left.d_{\xi}^{2} \phi\left(x_{*}, \xi_{*}\right)\left(N_{x_{*}}\left(\xi_{*}\right) \backslash 0 \times \cdot\right)\right|_{T_{\xi_{*}} S\left(x_{*}\right)} \quad \text { is of full rank, }
$$

where $N_{x_{*}}\left(\xi_{*}\right)$ denotes the kernel of $d_{\xi} \phi\left(x_{*}, \xi_{*}\right)$ and $S\left(x_{*}\right)$ is the set of all vectors $\xi \in T_{x_{*}}^{*} \mathbb{R}^{d}$ such that $\operatorname{det} d_{\xi} \phi\left(x_{*}, \xi\right)=0$. Indeed, in that case, it is shown in [35] that the canonical relation associated with the operator germ $\mathfrak{N}_{2, \xi_{*}}$ is locally a canonical graph and hence $\mathfrak{N}_{2, \xi_{*}}$ is bounded from $L^{2}\left(\Omega_{\epsilon_{0}}, \mathbb{R}^{2 d}\right)$ to $H^{\frac{d}{2}}\left(U\left(x_{*}\right), \mathbb{R}^{2 d}\right)$ for some neighborhood $U\left(x_{*}\right)$ of $x_{*}$. Note that for $d \geq 3, H^{\frac{d}{2}}\left(U\left(x_{*}\right), \mathbb{R}^{2 d}\right)$ is compactly embedded in $H^{1}\left(U\left(x_{*}\right), \mathbb{R}^{2 d}\right)$, so $\mathfrak{N}_{2, \xi_{*}}$ is compact from $L^{2}\left(\Omega_{\epsilon_{0}}, \mathbb{R}^{2 d}\right)$ to $H^{1}\left(U\left(x_{*}\right), \mathbb{R}^{2 d}\right)$ and we can conclude that $\xi_{*}$ is fold-regular.

The set of fold-regular vectors contains more elements than those which satisfy the graph condition (49). In fact, let $\mathcal{C} \subset T^{*} \Omega \times T^{*} \Omega$ be the canonical relation associated with the operator germ $\mathfrak{N}_{2, \xi_{*}}$ defined in Lemma 6.3. We can show that $\mathcal{C}$ is homogeneous and $\mathcal{C} \subset\left(T^{*} \Omega \backslash 0\right) \times\left(T^{*} \Omega \backslash 0\right)$. By the main result in [12, $\mathfrak{N}_{2, \xi_{*}}$ is bounded from $L^{2}\left(\Omega_{\epsilon_{0}}, \mathbb{R}^{2 d}\right)$ to $H^{\frac{d}{2}-\frac{1}{3}}\left(U\left(x_{*}\right), \mathbb{R}^{2 d}\right)$ for some neighborhood $U\left(x_{*}\right)$ of $x_{*}$, if the only singularity of the projection of $\mathcal{C}$ to its first or second component at the point associated with $\left(x_{*}, \xi_{*}\right)$ is fold or cusp. Since $H^{\frac{d}{2}-\frac{1}{3}}\left(U\left(x_{*}\right), \mathbb{R}^{2 d}\right)$ is compactly embedded in $H^{1}\left(U\left(x_{*}\right), \mathbb{R}^{2 d}\right)$, we see that $\mathfrak{N}_{2, \xi_{*}}$ is compact from $L^{2}\left(\Omega_{\epsilon_{0}}, \mathbb{R}^{2 d}\right)$ to $H^{1}\left(U\left(x_{*}\right), \mathbb{R}^{2 d}\right)$ and hence $\xi_{*}$ is fold-regular.

Remark 6.1. In dimension $d=2$, the set of fold-regular vectors is generally empty. Indeed, for a fold vector $\xi_{*}$, the operator germ $\mathfrak{N}_{2, \xi_{*}}$ is a FIO of order -1 , and hence the best estimate is that it is bounded from $L^{2}\left(\Omega_{\epsilon_{0}}, \mathbb{R}^{2 d}\right)$ to $H^{1}\left(U\left(x_{*}\right), \mathbb{R}^{2 d}\right)$ for some neighborhood $U\left(x_{*}\right)$ of $x_{*}$. 
We conclude this subsection with the following criterion for a fold-regular point.

Lemma 6.6. If for any $\theta \in S_{x}^{*} \mathbb{R}^{d}$, there exists $\xi \in S_{x}^{*} \mathbb{R}^{d}$ such that $\xi \perp \theta$ and that either no caustics or only fold caustics with condition (49) satisfied exist along the ray $\{\phi(x, t \xi):-T \leq t \leq T\}$, then the point $x$ is fold-regular.

6.4. Proof of Theorem 3.2, We prove Theorem 3.2 in this subsection. The proof can be divided into two major stages: in the first stage, we present some preliminaries and construct a cut-off function $\alpha \in C_{0}^{\infty}\left(S_{-}^{*} \Gamma\right)$ which selects a complete set of geodesics with only fold-regular caustics, see Lemma 6.7 in the second stage, we study the normal operator $\mathfrak{N}_{\alpha}=\mathfrak{I}_{\alpha}^{\dagger} \mathfrak{I}_{\alpha}$, see Lemmas 6.8 and 6.9. Theorem 3.2 is then a direct consequence of Lemmas 6.8 and 6.9.

We now present some preliminaries that are necessary for the construction of $\alpha$. Let $x_{*}$ be a fold-regular point with the compact subset $\mathcal{Z}\left(x_{*}\right) \subset S_{x_{*}}^{*} \mathbb{R}^{d}$ in Definition 3.4. Denote $\mathcal{C}_{\epsilon_{2}, T} \mathcal{Z}\left(x_{*}\right)=\left\{r \xi ; \xi \in \mathcal{Z}\left(x_{*}\right), r \in \mathbb{R}\right.$ and $\left.\epsilon_{2} \leq|r| \leq T\right\}$. For each $\xi_{*} \in$ $\mathcal{C}_{\epsilon_{2}, T} \mathcal{Z}\left(x_{*}\right)$, by Lemma 6.2 and Lemma 6.3. there exist a neighborhood $U\left(x_{*}, \xi_{*}\right)$ of $x_{*}$ and a neighborhood $B\left(x_{*}, \xi_{*}\right)$ of $\left(x_{*}, \xi_{*}\right)$ such that for any $\chi \in C_{0}^{\infty}\left(B\left(x_{*}, \xi_{*}\right)\right)$ the following operator:

$$
\mathfrak{N}_{2, \xi_{*}} f(x)=\int_{T_{x}^{*} \Omega} W(x, \xi) f(\phi(x, \xi))\left(1-\chi_{\epsilon_{2}}(|\xi|)\right) \cdot \chi(x, \xi) d \sigma_{x}(\xi)
$$

is compact from $H^{s}\left(\Omega_{\epsilon_{0}}, \mathbb{R}^{2 d}\right)$ to $H^{s+1}\left(U\left(x_{*}, \xi_{*}\right), \mathbb{R}^{2 d}\right)$. Let $B_{0}\left(x_{*}, \xi_{*}\right)$ be another neighborhood of $\left(x_{*}, \xi_{*}\right)$ in $\mathbb{R}^{2 d}$ such that $B_{0}\left(x_{*}, \xi_{*}\right) \Subset B\left(x_{*}, \xi_{*}\right)$. Since $\mathcal{C}_{\epsilon_{2}, T} \mathcal{Z}\left(x_{*}\right)$ is compact, there exists a finite number of $\xi_{*}$ 's in $\mathcal{C}_{\epsilon_{2}, T} \mathcal{Z}\left(x_{*}\right)$, say $\xi_{1}, \xi_{2}, \ldots, \xi_{M}$, such that

$$
\mathcal{C}_{\epsilon_{2}, T} \mathcal{Z}\left(x_{*}\right) \subset \bigcup_{j=1}^{M} B_{0}\left(x_{*}, \xi_{j}\right) .
$$

We can then find smooth functions $\chi_{1}, \chi_{2}, \ldots, \chi_{M}$ with $\operatorname{supp} \chi_{j} \subset B\left(x_{*}, \xi_{j}\right)$ for each $j$ such that

$$
\sum_{j=1}^{M} \chi_{j}(x, \xi)=1 \quad \text { for all }(x, \xi) \in \bigcup_{j=1}^{M} B_{0}\left(x_{*}, \xi_{j}\right) .
$$

Denote by $\mathcal{A}_{0}$, the greatest connected open symmetric subset in $\bigcup_{j=1}^{M} B_{0}\left(x_{*}, \xi_{j}\right)$ which contains $\mathcal{C}_{\epsilon_{2}, T} \mathcal{Z}\left(x_{*}\right)$. Here and after, we say that a set $\mathcal{B}$ in $\mathbb{R}^{2 d}$ is symmetric if $(x, \xi) \in \mathcal{B}$ implies that $(x,-\xi) \in \mathcal{B}$. Define

$$
\mathcal{A}_{\epsilon}=\left\{(x, \xi) \in \mathbb{R}^{2 d}:\left|x-x_{*}\right| \leq \epsilon, \epsilon_{2} \leq|\xi| \leq T\right\}
$$

for each $\epsilon>0$. It is clear that $\mathcal{A}_{\epsilon}$ is compact in $\mathbb{R}^{2 d}$, so is the set $\mathcal{A}_{\epsilon} \backslash \mathcal{A}_{0}$.

Lemma 6.7. There exists $\epsilon_{3}>0$ and $\alpha \in C_{0}^{\infty}\left(S_{-}^{*} \Gamma\right)$ such that the following two conditions are satisfied:

$$
\begin{array}{ll}
\alpha\left(x_{0}, \xi_{0}\right)=1 & \text { for all }\left(x_{0}, \xi_{0}\right) \in \tau \circ \beta\left(\mathcal{C}_{\epsilon_{2}, T} \mathcal{Z}\left(x_{*}\right)\right), \\
\alpha\left(x_{0}, \xi_{0}\right)=0 & \text { for all }\left(x_{0}, \xi_{0}\right) \in \tau \circ \beta\left(\mathcal{A}_{\epsilon_{3}} \backslash \mathcal{A}_{0}\right) .
\end{array}
$$

Proof. Note that both $\beta$ and $\tau$ are continuous. Since $\mathcal{C}_{\epsilon_{2}, T} \mathcal{Z}\left(x_{*}\right)$ and $\mathcal{A}_{\epsilon} \backslash \mathcal{A}_{0}$ are compact, so are the sets $\tau \circ \beta\left(\mathcal{C}_{\epsilon_{2}, T} \mathcal{Z}\left(x_{*}\right)\right)$ and $\tau \circ \beta\left(\mathcal{A}_{\epsilon} \backslash \mathcal{A}_{0}\right)$. We claim that there exists $\epsilon_{3}>0$ such that

$$
\tau \circ \beta\left(\mathcal{C}_{\epsilon_{2}, T} \mathcal{Z}\left(x_{*}\right)\right) \bigcap \tau \circ \beta\left(\mathcal{A}_{\epsilon} \backslash \mathcal{A}_{0}\right)=\emptyset
$$


for all $\epsilon \leq \epsilon_{3}$. Indeed, assume the contrary, then

$$
\tau \circ \beta\left(\mathcal{C}_{\epsilon_{2}, T} \mathcal{Z}\left(x_{*}\right)\right) \bigcap \tau \circ \beta\left(\mathcal{A}_{\epsilon} \backslash \mathcal{A}_{0}\right) \neq \emptyset
$$

for all $\epsilon>0$. Note that the collection of compact subsets $\tau \circ \beta\left(\mathcal{C}_{\epsilon_{2}, T} \mathcal{Z}\left(x_{*}\right)\right) \bigcap \tau \circ$ $\beta\left(\mathcal{A}_{\epsilon} \backslash \mathcal{A}_{0}\right)$ is decreasing with respect to $\epsilon$, so it satisfies the finite intersection property and we can thus conclude that

$$
\tau \circ \beta\left(\mathcal{C}_{\epsilon_{2}, T} \mathcal{Z}\left(x_{*}\right)\right) \bigcap_{\epsilon>0} \tau \circ \beta\left(\mathcal{A}_{\epsilon} \backslash \mathcal{A}_{0}\right) \neq \emptyset .
$$

But on the other hand, we can check that

$$
\begin{aligned}
& \bigcap_{\epsilon>0} \tau \circ \beta\left(\mathcal{A}_{\epsilon} \backslash \mathcal{A}_{0}\right)=\tau\left(\left(\mathcal{A}_{\epsilon} \backslash \mathcal{A}_{0}\right) \bigcap S_{x_{*}}^{*} \mathbb{R}^{d}\right), \\
& \tau \circ \beta\left(\mathcal{C}_{\epsilon_{2}, T} \mathcal{Z}\left(x_{*}\right)\right)=\tau\left(\mathcal{C}_{\epsilon_{2}, T} \mathcal{Z}\left(x_{*}\right) \bigcap S_{x_{*}}^{*} \mathbb{R}^{d}\right) .
\end{aligned}
$$

Using the fact that $\tau$ is injective on $S_{x_{*}}^{*} \mathbb{R}^{d}$ and $\mathcal{C}_{\epsilon_{2}, T} \mathcal{Z}\left(x_{*}\right) \subset \mathcal{A}_{0}$, we obtain

$$
\tau\left(\left(\mathcal{A}_{\epsilon} \backslash \mathcal{A}_{0}\right) \bigcap S_{x_{*}}^{*} \mathbb{R}^{d}\right) \bigcap \tau\left(\mathcal{C}_{\epsilon_{2}, T} \mathcal{Z}\left(x_{*}\right) \bigcap S_{x_{*}}^{*} \mathbb{R}^{d}\right)=\emptyset .
$$

Thus,

$$
\tau \circ \beta\left(\mathcal{C}_{\epsilon_{2}, T} \mathcal{Z}\left(x_{*}\right)\right) \bigcap_{\epsilon>0} \tau \circ \beta\left(\mathcal{A}_{\epsilon} \backslash \mathcal{A}_{0}\right)=\emptyset .
$$

This contradiction completes the proof of our claim.

Now, we have

$$
\tau \circ \beta\left(\mathcal{C}_{\epsilon_{2}, T} \mathcal{Z}\left(x_{*}\right)\right) \bigcap \tau \circ \beta\left(\mathcal{A}_{\epsilon_{3}} \backslash \mathcal{A}_{0}\right)=\emptyset .
$$

By decreasing $\epsilon_{3}$ if necessary, we may assume that

$$
\left\{x:\left|x-x_{*}\right| \leq \epsilon_{3}\right\} \subset \pi\left(\mathcal{A}_{0}\right) .
$$

Since both the sets $\tau \circ \beta\left(\mathcal{C}_{\epsilon_{2}, T} \mathcal{Z}\left(x_{*}\right)\right)$ and $\tau \circ \beta\left(\mathcal{A}_{\epsilon_{3}} \backslash \mathcal{A}_{0}\right)$ are compact in $S_{-}^{*} \Gamma$, we can find $\alpha \in C_{0}^{\infty}\left(S_{-}^{*} \Gamma\right)$ as desired. This concludes the proof of the lemma.

The construction of $\alpha$ above completes the first stage of the proof of Theorem 3.2 we are now at the second stage. We define the truncated geodesic X-ray transform $\mathfrak{I}_{\alpha} f$ as in (23) or (24). By replacing the weight $\Phi$ with the new one $\alpha^{\sharp} \cdot \Phi$, we obtain $\mathfrak{N}_{\alpha}, \mathfrak{N}_{1, \alpha}$ and $\mathfrak{N}_{2, \alpha}$ from the corresponding formulas of $\mathfrak{N}, \mathfrak{N}_{1}$ and $\mathfrak{N}_{2}$. It is clear that Lemmas 6.2 and 6.3 still hold with the new weight.

Lemma 6.8. There exist a neighborhood $U\left(x_{*}\right)$ of $x_{*}$ and a smoothing operator $\Re$ from $\mathcal{E}^{\prime}\left(\Omega, \mathbb{R}^{2 d}\right)$ into $C^{\infty}\left(\overline{U\left(x_{*}\right)}, \mathbb{R}^{2 d}\right)$, such that for for any $s \in \mathbb{R}$ and any neighborhood $U_{0}\left(x_{*}\right)$ of $x_{*}$ with $U_{0}\left(x_{*}\right) \Subset U\left(x_{*}\right)$, the following estimate holds:

$$
\|f\|_{H^{s}\left(U_{0}\left(x_{*}\right), \mathbb{R}^{2 d}\right)} \lesssim\left\|\mathfrak{N}_{1, \alpha} f\right\|_{H^{s+1}\left(U\left(x_{*}\right), \mathbb{R}^{2 d}\right)}+\|\mathfrak{R} f\|_{H^{s}\left(\Omega, \mathbb{R}^{2 d}\right)} .
$$

Proof. We first show that $\mathfrak{N}_{1, \alpha}$ is an elliptic $\Psi$ DO. Indeed, as in Lemma 6.1, $\mathfrak{N}_{1, \alpha}$ is a $\Psi$ DO of order -1 from $C_{0}^{\infty}\left(\tilde{U}\left(x_{*}\right), \mathbb{R}^{2 d}\right)$ to $\mathcal{D}^{\prime}\left(\tilde{U}\left(x_{*}\right), \mathbb{R}^{2 d}\right)$ with principle symbol

$$
\sigma_{p}\left(\mathfrak{N}_{1}\right)(x, \xi)=2 \pi \cdot \int_{S_{x}^{*} \Omega} \delta(\langle\xi, \theta\rangle)\left|\alpha^{\sharp}\left(x_{*}, \theta\right)\right|^{2} \Phi^{\dagger}(x, \theta) \cdot \Phi(x, \theta) d \sigma_{x}(\theta) .
$$


By the construction of $\alpha$, for any $\xi \in S_{x_{*}}^{*} \mathbb{R}^{d}$, we have $\alpha^{\sharp}\left(x_{*}, \theta\right)=1$ for some $\theta \in S_{x_{*}}^{*} \mathbb{R}^{d}$ with $\theta \perp \xi$. Thus

$$
\sigma_{p}\left(\mathfrak{N}_{1, \alpha}\right)\left(x_{*}, \xi\right)=2 \pi \cdot \int_{\theta \in S_{x_{*}^{*}} \mathbb{R}^{d}, \theta \perp \xi}\left|\alpha^{\sharp}\left(x_{*}, \theta\right)\right|^{2} \Phi^{\dagger}\left(x_{*}, \theta\right) \cdot \Phi\left(x_{*}, \theta\right) d \sigma_{x_{*}}(\theta)>0
$$

in the sense of symmetric positive definite matrix. By continuity, we can find a neighborhood $U\left(x_{*}\right) \subset \tilde{U}\left(x_{*}\right)$ of $x_{*}$ such that $\sigma_{p}\left(\mathfrak{N}_{1, \alpha}\right)(x, \xi)>0$ for all $x \in U\left(x_{*}\right)$ and $\xi \in S_{x}^{*} \mathbb{R}^{d}$. Thus we can conclude that $\mathfrak{N}_{1, \alpha}$ is an elliptic $\Psi$ DO of order -1 from $C_{0}^{\infty}\left(U\left(x_{*}\right), \mathbb{R}^{2 d}\right)$ to $\mathcal{D}^{\prime}\left(U\left(x_{*}\right), \mathbb{R}^{2 d}\right)$. By the standard argument, we can conclude that Lemma 6.8 holds.

We now study the operator $\mathfrak{N}_{2, \alpha}$.

Lemma 6.9. There exists a small neighborhood of $x_{*}$, say $U\left(x_{*}\right)$, such that the operator $\mathfrak{N}_{2, \alpha}: \mathcal{E}^{\prime}\left(\Omega, \mathbb{R}^{2 d}\right) \rightarrow \mathcal{D}^{\prime}\left(U\left(x_{*}\right), \mathbb{R}^{2 d}\right)$ is compact from $H^{s}\left(\Omega_{\epsilon_{0}}, \mathbb{R}^{2 d}\right)$ to $H^{s+1}\left(U\left(x_{*}\right), \mathbb{R}^{2 d}\right)$.

Proof. Recall that $\mathfrak{N}_{2, \alpha}$ has the following representation:

$$
\mathfrak{N}_{2, \alpha} f(x)=\int_{T_{x}^{*} \Omega} W_{\alpha}(x, \xi) f(\phi(x, \xi))\left(1-\chi_{\epsilon_{2}}(|\xi|)\right) \cdot d \sigma_{x}(\xi),
$$

where

$$
\begin{aligned}
W_{\alpha}(x, \xi)= & \frac{1}{|\xi|^{d-1}}|\alpha \circ \tau \circ \beta(x, \xi)|^{2} \Phi^{\dagger} \circ \beta(x, \xi) \cdot \Phi \circ \beta \circ \mathcal{H}(x, \xi) \\
& +\frac{1}{|\xi|^{d-1}}|\alpha \circ \tau \circ \beta(x,-\xi)|^{2} \Phi^{\dagger} \circ \beta(x,-\xi) \cdot \Phi \circ \beta \circ \mathcal{H}^{-1}(x,-\xi) .
\end{aligned}
$$

By (51) and the fact that $\mathcal{A}_{0}$ is symmetric, we see that supp $W_{\alpha} \subset \mathcal{A}_{0}$ for all $x$ with $\left|x-x_{*}\right| \leq \epsilon_{3}$. by

Now, let $\chi_{j}$ 's be as in the first stage. Define $\mathfrak{N}_{2, j}: \mathcal{E}^{\prime}\left(\Omega, \mathbb{R}^{2 d}\right) \rightarrow \mathcal{D}^{\prime}\left(U\left(x_{*}, \xi_{j}\right), \mathbb{R}^{2 d}\right)$

$$
\mathfrak{N}_{2, j} f(x)=\int_{T_{x}^{*} \Omega} W_{\alpha}(x, \xi) f(\phi(x, \xi))\left(1-\chi_{\epsilon_{2}}(|\xi|)\right) \cdot \chi_{j}(x, \xi) d \sigma_{x}(\xi) .
$$

Let $U\left(x_{*}\right)=\bigcap_{j=1}^{M}\left(U\left(x_{*}, \xi_{j}\right)\right) \bigcap\left\{x:\left|x-x_{*}\right|<\epsilon_{3}\right\}$. Then $U\left(x_{*}\right)$ is a neighborhood of $x_{*}$ and each $\mathfrak{N}_{2, j}$ is compact from $H^{s}\left(\Omega_{\epsilon_{0}}, \mathbb{R}^{2 d}\right)$ into $H^{s+1}\left(U\left(x_{*}\right), \mathbb{R}^{2 d}\right)$.

We claim that $\mathfrak{N}_{2, \alpha}=\sum_{j=1}^{M} \mathfrak{N}_{2, j}$ when both sides are viewed as operators from $\mathcal{E}^{\prime}\left(\Omega, \mathbb{R}^{2 d}\right)$ to $\mathcal{D}^{\prime}\left(U\left(x_{*}\right), \mathbb{R}^{2 d}\right)$. Indeed, for any $f \in C_{0}^{\infty}\left(\Omega, \mathbb{R}^{2 d}\right)$, since $\sum_{j=1}^{M} \chi_{j}=1$ on $\mathcal{A}_{0}$ and supp $W_{\alpha} \subset \mathcal{A}_{0}$, we have

$W_{\alpha}(x, \xi) f(\phi(x, \xi))\left(1-\chi_{\epsilon_{2}}(|\xi|)\right)=W_{\alpha}(x, \xi) f(\phi(x, \xi))\left(1-\chi_{\epsilon_{2}}(|\xi|)\right) \cdot\left(\sum_{j=1}^{M} \chi_{j}(x, \xi)\right)$

for all $x \in U\left(x_{*}\right)$. Thus $\mathfrak{N}_{2, \alpha} f=\sum_{j=1}^{M} \mathfrak{N}_{2, j} f$ and the claim follows. This completes the proof of the lemma.

Finally, note that $\mathfrak{N}_{\alpha}=\mathfrak{N}_{1, \alpha}+\mathfrak{N}_{2, \alpha}$. Theorem 3.2 follows from Lemmas 6.8 and 6.9 


\section{SEnSitivity ANALYSis OF RECOVERING THE VELOCITy FIELD FROM THE DDTN MAP}

In this section, we prove Theorem 3.3 on the sensitivity of the inverse problem of recovering the velocity field from the DDtN map. We first present a lemma which is a direct consequence of Theorem 3.1 and Lemma 3.3 .

Lemma 7.1. Let $c$ and $\tilde{c}$ be two velocity field in $\mathfrak{A}\left(\epsilon_{0}, \Omega, M_{0}, T\right)$, and let $f$ be as in (14). Then there exists $\delta>0$ such that if $\left\|\Lambda_{\tilde{c}}-\Lambda_{c}\right\|_{H_{0}^{1}\left[0,3 \epsilon_{1} / 4\right] \times \Gamma \rightarrow L^{2}\left(\left[0, T+\epsilon_{1}\right] \times \Gamma\right)} \leq \delta$, then

$$
\|\Im f\|_{L^{\infty}\left(S_{-}^{*} \Gamma, \mathbb{R}^{2 d}\right)} \leq C\|f\|_{C^{1}\left(\Omega, \mathbb{R}^{2 d}\right)}^{2}
$$

for constant $C>0$ depending $M_{0}$.

Proof of Theorem 3.3 The proof is divided into the following six steps.

Step 1. Since $c$ is fold-regular, for each $x \in \bar{\Omega}_{\epsilon_{0}}$, by Theorem 3.2 , there exist a neighborhood $U(x)$ of $x$, a smooth cut-off function $\alpha \in C_{0}^{\infty}\left(S_{-}^{*} \Gamma\right)$ and a smoothing operator $\mathfrak{R}$ such that for any $U_{0}(x) \Subset U(x)$ the following estimate holds:

$$
\|f\|_{L^{2}\left(U_{0}(x), \mathbb{R}^{2 d}\right)} \lesssim\left\|\mathfrak{N}_{\alpha} f\right\|_{H^{1}\left(U(x), \mathbb{R}^{2 d}\right)}+\left\|\mathfrak{N}_{2, \alpha} f\right\|_{H^{1}\left(\Omega, \mathbb{R}^{2 d}\right)}+\|\Re f\|_{H^{1}\left(\Omega, \mathbb{R}^{2 d}\right)}
$$

for all $f \in L^{2}\left(\Omega_{\epsilon_{0}}, \mathbb{R}^{2 d}\right)$. Moreover, both $\mathfrak{N}_{2, \alpha}$ and $\mathfrak{R}$ are compact from $L^{2}\left(\Omega_{\epsilon_{0}}, \mathbb{R}^{2 d}\right)$ to $H^{1}\left(U(x), \mathbb{R}^{2 d}\right)$.

We now fix a neighborhood $U_{0}(x) \Subset U(x)$ of $x$ for each $x$. Note that $\bar{\Omega}_{\epsilon_{0}}$ is compact, there exists a finite number of points, say $x_{1}, x_{2}, \ldots x_{M}$ such that $\bar{\Omega}_{\epsilon_{0}} \subset \bigcup_{j=1}^{M} U_{0}\left(x_{j}\right)$. Let $\mathfrak{N}_{\alpha_{j}}$ be the operator associated with each point $x_{j}$.

Step 2. Denote by $H$ the Hilbert space $\prod_{j=1}^{M} H^{1}\left(U\left(x_{j}\right), \mathbb{R}^{2 d}\right)$. We consider the following three operators:

$$
\begin{aligned}
T f & =\left(\mathfrak{N}_{\alpha_{1}} f, \mathfrak{N}_{\alpha_{2}} f, \ldots, \mathfrak{N}_{\alpha_{M}} f\right), \\
T_{1} f & =\left(\mathfrak{N}_{2, \alpha_{1}} f, \mathfrak{N}_{2, \alpha_{2}} f, \ldots, \mathfrak{N}_{2, \alpha_{M}} f\right), \\
T_{2} f & =\left(\mathfrak{R}_{\alpha_{1}} f, \mathfrak{R}_{\alpha_{2}} f, \ldots, \mathfrak{R}_{\alpha_{M}} f\right) .
\end{aligned}
$$

It is clear that all three operators are bounded from $L^{2}\left(\Omega_{\epsilon_{0}}\right)$ to $H$. Moreover, $T_{1}$ and $T_{2}$ are also compact and the following estimate holds:

$$
\|f\|_{L^{2}\left(\Omega, \mathbb{R}^{2 d}\right)} \lesssim\|T f\|_{H}+\left\|T_{1} f\right\|_{H}+\left\|T_{2} f\right\|_{H} .
$$

Step 3. Let $\mathfrak{L}_{0} \subset L^{2}\left(\Omega_{\epsilon_{0}}, \mathbb{R}^{2 d}\right)$ be the kernel of $T$. We claim that $\mathfrak{L}_{0} \subset$ $L^{2}\left(\Omega_{\epsilon_{0}}, \mathbb{R}^{2 d}\right)$ is of finite dimension. We prove by contradiction. Assume the contrary, then there exists an infinity number of orthogonal vectors in $\mathfrak{L}_{0} \subset L^{2}\left(\Omega_{\epsilon_{0}}, \mathbb{R}^{2 d}\right)$, say, $e_{1}, e_{2}, \ldots$, such that $\left\|e_{j}\right\|_{L^{2}\left(\Omega_{\epsilon_{0}}, \mathbb{R}^{2 d}\right)}=1$ and $T e_{j}=0$ for all $j \in \mathbb{N}$. Since the sequence $\left\{e_{j}\right\}_{j=1}^{\infty}$ is bounded in $L^{2}\left(\Omega_{\epsilon_{0}}, \mathbb{R}^{2 d}\right)$ and the operators $T_{1}$ and $T_{2}$ are compact, we can find a subsequence, still denoted by $\left\{e_{j}\right\}_{j=1}^{\infty}$, such that both the sequences $\left\{T_{1} e_{j}\right\}_{j=1}^{\infty}$ and $\left\{T_{2} e_{j}\right\}_{j=1}^{\infty}$ are Cauchy in $H$. By applying Inequality (55) to the vectors $e_{i}-e_{j}$ and recalling that $T\left(e_{i}-e_{j}\right)=0$, we conclude that the sequence $\left\{e_{j}\right\}_{j=1}^{\infty}$ is also Cauchy in $L^{2}\left(\Omega_{\epsilon_{0}}, \mathbb{R}^{2 d}\right)$. This contradicts to the fact that $\left\|e_{i}-e_{j}\right\|_{L^{2}\left(\Omega_{\epsilon_{0}}, \mathbb{R}^{2 d}\right)}>1$ for all $i \neq j$. This contradiction proves the claim. that

Step 4. Denote by $\mathfrak{L}_{0}^{\perp}$ the orthogonal space to $\mathfrak{L}_{0}$ in $L^{2}\left(\Omega_{\epsilon_{0}}, \mathbb{R}^{2 d}\right)$. We claim

$$
\|f\|_{L^{2}\left(\Omega_{\epsilon_{0}}, \mathbb{R}^{2 d}\right)} \lesssim\|T f\|_{H} \quad \text { for all } f \in \mathfrak{L}_{0}^{\perp}
$$


Indeed, assume the contrary, there exists a sequence $\left\{f_{n}\right\}_{n=1}^{\infty} \subset \mathfrak{L}^{\perp}$ such that $\left\|f_{n}\right\|_{L^{2}\left(\Omega_{\epsilon_{0}}, \mathbb{R}^{2 d}\right)}=1$ and $\left\|T f_{n}\right\|_{H} \leq \frac{1}{n}$ for all $n$. By the same argument as in Step 3, we can find a subsequence, still denoted by $\left\{e_{j}\right\}_{j=1}^{\infty}$, such that both the sequences $\left\{T_{1} e_{j}\right\}_{j=1}^{\infty}$ and $\left\{T_{2} e_{j}\right\}_{j=1}^{\infty}$ are Cauchy in $H$. By Inequality (55) and the fact that $\left\|T f_{n}\right\|_{H} \leq \frac{1}{n}$, we can conclude that $\left\{f_{n}\right\}_{n=1}^{\infty}$ is also Cauchy in $L^{2}\left(\Omega_{\epsilon_{0}}, \mathbb{R}^{2 d}\right)$. Let $f_{0}=\lim _{n \rightarrow \infty} f_{n}$, then $\left\|T f_{0}\right\|_{H}=\lim _{n \rightarrow \infty}\left\|T f_{n}\right\|_{H}=0$. This implies that $f_{0} \in \mathfrak{L}_{0}$. However, note that $\mathfrak{L}_{0}^{\perp}$ is closed, as the limit of a sequence of functions in $\mathfrak{L}_{0}^{\perp}, f_{0}$ must belong to $\mathfrak{L}_{0}^{\perp}$. Therefore, we see that $f_{0}=0$. But this contradicts to the fact that $\left\|f_{0}\right\|_{L^{2}\left(\Omega_{\epsilon_{0}}, \mathbb{R}^{2 d}\right)}=\lim _{n \rightarrow \infty}\left\|f_{n}\right\|_{L^{2}\left(\Omega_{\epsilon_{0}}, \mathbb{R}^{2 d}\right)}=1$. The claim is proved.

Step 5. From now on, let $f$ be as in (14). We claim that

$$
\|T f\|_{H} \lesssim\|f\|_{H^{\frac{15}{2}\left(\Omega, \mathbb{R}^{2 d}\right)}}^{\frac{2}{5}} \cdot\|f\|_{L^{2}\left(\Omega, \mathbb{R}^{2 d}\right)} .
$$

Indeed, for each $\mathfrak{I}_{\alpha_{j}}$, by Lemma 17.1, we have $\left\|\mathfrak{I}_{\alpha_{j}} f\right\|_{L^{\infty}\left(S_{-}^{*} \Gamma\right)} \leq C_{1}\|f\|_{C^{1}}^{2}$. Apply $\mathfrak{I}_{\alpha_{j}}^{\dagger}$ to both sides and use the fact that $\mathfrak{I}_{\alpha_{j}}^{\dagger}$ is bounded from $L^{2}$ to $L^{2}$ (see [24]); we obtain

$$
\left\|\mathfrak{N}_{\alpha_{j}} f\right\|_{L^{2}\left(\Omega, \mathbb{R}^{2 d}\right)} \lesssim\|f\|_{C^{1}\left(\Omega, \mathbb{R}^{2 d}\right)}^{2} .
$$

Then,

$$
\begin{aligned}
& \left\|\mathfrak{N}_{\alpha_{j}} f\right\|_{H^{1}\left(U\left(x_{j}\right), \mathbb{R}^{2 d}\right)} \\
& \lesssim\left\|\mathfrak{N}_{\alpha_{j}} f\right\|_{H^{3}\left(U\left(x_{j}\right), \mathbb{R}^{2 d}\right)}^{\frac{1}{3}} \cdot\left\|\mathfrak{N}_{\alpha} f\right\|_{L^{2}\left(\Omega, \mathbb{R}^{2 d}\right)}^{\frac{2}{3}} \quad \text { (by interpolation inequality) } \\
& \lesssim\left\|\mathfrak{N}_{\alpha_{j}} f\right\|_{H^{3}\left(U\left(x_{j}\right), \mathbb{R}^{2 d}\right)}^{\frac{1}{3}} \cdot\|f\|_{C^{1}\left(\Omega, \mathbb{R}^{2 d}\right)}^{\frac{4}{3}} \quad \text { (by }(\underline{57}) \\
& \lesssim\|f\|_{H^{3}\left(\Omega, \mathbb{R}^{2 d}\right)}^{\frac{1}{3}} \cdot\|f\|_{C^{1}\left(\Omega, \mathbb{R}^{2 d}\right)}^{\frac{4}{3}} \quad \text { (by Lemmas 6.8, 6.9) } \\
& \lesssim\|f\|_{H^{3}\left(\Omega, \mathbb{R}^{2 d}\right)}^{\frac{1}{3}} \cdot\|f\|_{H^{3}\left(\Omega, \mathbb{R}^{2 d}\right)}^{\frac{4}{3}} \quad \text { (by interpolation inequality) } \\
& =\|f\|_{H^{3}\left(\Omega, \mathbb{R}^{2 d}\right)}^{\frac{5}{3}} \\
& \lesssim\|f\|_{H^{\frac{15}{2}}\left(\Omega, \mathbb{R}^{2 d}\right)}^{\frac{2}{5}} \cdot\|f\|_{L^{2}\left(\Omega, \mathbb{R}^{2 d}\right)} . \quad \text { (by interpolation inequality) }
\end{aligned}
$$

It follows that

$$
\|T f\|_{H}=\sum_{j=1}^{M}\left\|\mathfrak{N}_{\alpha_{j}} f\right\|_{H^{1}\left(U\left(x_{j}\right), \mathbb{R}^{2 d}\right)} \lesssim\|f\|_{H^{\frac{15}{2}}\left(\Omega, \mathbb{R}^{2 d}\right)}^{\frac{2}{5}} \cdot\|f\|_{L^{2}\left(\Omega, \mathbb{R}^{2 d}\right)} .
$$

This finishes the proof of our claim.

Step 6 . Denote by $\mathfrak{L}$ the projection of $\mathfrak{L}_{0}$ from $L^{2}\left(\Omega, \mathbb{R}^{2 d}\right)$ to the space $L^{2}\left(\Omega, \mathbb{R}^{d}\right)$ by taking the last three components. Note that the first three components of $f$ are zero; see (14). Thus the condition $\nabla\left(\ln c^{2}-\ln \tilde{c}^{2}\right) \perp \mathfrak{L}$ implies that $f \in \mathfrak{L}_{0}^{\perp}$. Consequently, Inequality (56) holds. Combining this with (58), we see that

$$
\|f\|_{L^{2}\left(\Omega, \mathbb{R}^{2 d}\right)} \lesssim\|f\|_{H^{\frac{15}{2}}\left(\Omega, \mathbb{R}^{2 d}\right)}^{\frac{2}{5}} \cdot\|f\|_{L^{2}\left(\Omega, \mathbb{R}^{2 d}\right)} .
$$

Therefore, we must have $f=0$ for $\|f\|_{H^{\frac{15}{2}}\left(\Omega, \mathbb{R}^{2 d}\right)}^{\frac{2}{5}}$ sufficiently small. Finally, note that $\|f\|_{H^{\frac{15}{2}}\left(\Omega, \mathbb{R}^{2 d}\right)} \lesssim\|c-\tilde{c}\|_{H^{\frac{17}{2}}(\Omega)}$ and that both $c$ and $\tilde{c}$ vanishes near the boundary, we conclude that $f=0$ implies $c=\tilde{c}$. This completes the proof of the theorem. 


\section{ACKnowledgements}

We thank the anonymous referees for many useful suggestions which have significantly improved the presentation of the paper.

\section{Appendix}

8.1. Linearization of the ODE system. Given the following ODE system:

$$
\dot{y}=f(y), \quad y=y_{o},
$$

where $f \in C^{1}\left(\mathbb{R}^{d}, \mathbb{R}^{d}\right)$. We consider the perturbed the system

$$
\dot{y}_{\epsilon}=f_{\epsilon}\left(y_{\epsilon}\right), \quad y_{\epsilon}=y_{o}
$$

where $f_{\epsilon}=f+\epsilon g$ with $g \in C^{1}\left(\mathbb{R}^{d}\right)$. We formally write $y_{\epsilon}(t)=y(t)+r(t)=$ $y(t)+\epsilon \phi(t)+r_{1}(t)$, where $\phi(0)=r(0)=r_{1}(0)=0$. By substituting $y_{\epsilon}(t)=$ $y(t)+\epsilon \phi(t)+r_{1}(t)$ into the perturbed system, we can derive that $\phi$ satisfies the following equation:

$$
\dot{\phi}(t)=\frac{\partial f}{\partial y}(y(t)) \cdot \phi(t)+g(y(t)), \quad \phi(0)=0 .
$$

By Grownwall's inequality, we can show that $|r(t)| \leq C \epsilon$ and $\left|r_{1}(t)\right| \leq C \epsilon^{2}$, where $C$ is a constant depending on $\|f\|_{C^{2}}+\|g\|_{C^{1}}$.

We solve Equation (59) as follows. Let $A(t)=\frac{\partial f}{\partial y}(y(t))$. Let $\Phi(t)$ and $\Psi(t)$ be the solution to the following ODE system:

$$
\begin{aligned}
& \dot{\Phi}(t)=-\Phi(t) A(t), \quad \Phi(0)=I d ; \\
& \dot{\Psi}(t)=A(t) \Psi(t), \quad \Psi(0)=I d .
\end{aligned}
$$

A straightforward calculation shows that $\Phi(t) \Psi(t) \equiv \Phi(0) \Psi(0)=I d$. Moreover,

$$
\phi(t)=\Phi(t)^{-1} \int_{0}^{t} \Phi(s) g(y(s)) d s .
$$

8.2. Proof of Lemma 4.2 and Lemma 4.4. Proof of 4.2. We only show (30), since (31) follows in a similar way. For simplicity, denote $D=\left(3 \epsilon_{1} / 4, t_{1}+\epsilon_{1} / 2\right) \times$ $U\left(x_{1}\right)$. We first show that

$$
\hat{g}(t, y, \lambda)=\hat{g}_{*}(t, y, \lambda)+O\left(\frac{1}{\sqrt{\lambda}}\right) \text { in } L^{2}(D) .
$$

Indeed, by direct calculation,

$$
\hat{g}(t, y, \lambda)-\hat{g}_{*}(t, y, \lambda)=\left(a(t)-a\left(t_{1}\right)\right) e^{i \lambda \hat{\tau}_{*}}+a(t)\left(e^{i \lambda \hat{\tau}}-e^{i \lambda \hat{\tau}_{*}}\right) .
$$

It suffices to show that

$$
\begin{aligned}
& R_{1}:=\left\|\left(a(t)-a\left(t_{1}\right)\right) e^{i \lambda \hat{\tau}_{*}}\right\|_{L^{2}(D)} \lesssim \frac{1}{\sqrt{\lambda}}, \\
& R_{2}:=\left\|a(t)\left(e^{i \lambda \hat{\tau}}-e^{i \lambda \hat{\tau}_{*}}\right)\right\|_{L^{2}(D)} \lesssim \frac{1}{\sqrt{\lambda}} .
\end{aligned}
$$

We first estimate $R_{1}$. By Lemma 3.1 in [5], we have $|a(t)| \approx \lambda^{\frac{d}{4}}$. By Equation (27), we further derive that $|\dot{a}(t)| \approx \lambda^{\frac{d}{4}}$, thus

$$
a(t)-a\left(t_{1}\right)=\int_{0}^{1} \dot{a}\left(t_{1}+s\left(t-t_{1}\right)\right) d s\left(t-t_{1}\right)=O\left(\lambda^{\frac{d}{4}}\right)\left|t-t_{1}\right| .
$$


Therefore,

$$
\left\|\left(a(t)-a\left(t_{1}\right)\right) e^{i \lambda \hat{\tau}_{*}}\right\|_{L^{2}(D)}^{2} \lesssim \int_{D} \lambda^{\frac{d}{2}}\left(t-t_{1}\right)^{2} e^{-\lambda\left(t-t_{1}, y\right) \Im \hat{M}\left(t_{1}\right)\left(t-t_{1}, y\right)^{\dagger}} d t d y \lesssim \frac{1}{\lambda} .
$$

This proves $R_{1} \lesssim \frac{1}{\sqrt{\lambda}}$.

We next estimate $R_{2}$. Write $\hat{\tau}=\hat{\tau}_{*}+\delta \hat{\tau}$, then $\delta \hat{\tau}=O\left(\left|\left(t-t_{1}, y\right)\right|^{3}\right)$ and hence $\left|1-e^{i \lambda \delta \hat{\tau}}\right| \lesssim \lambda \cdot O\left(\left|\left(t-t_{1}, y\right)\right|^{3}\right)$. It follows that

$$
\begin{aligned}
R_{2} & \leq \int_{D}\left|a(t) e^{i \lambda \hat{\tau}_{*}}\right|^{2} \cdot\left|1-e^{i \lambda \delta \hat{\tau}}\right| d t d y \\
& \lesssim \int_{D} \lambda^{\frac{d}{2}} \cdot \lambda \cdot\left|\left(t-t_{1}, y\right)\right|^{3} e^{-2 \lambda\left(t-t_{1}, y\right) \Im \hat{M}\left(t_{1}\right)\left(t-t_{1}, y\right)^{\dagger}} d t d y \lesssim \frac{1}{\lambda} .
\end{aligned}
$$

This completes the proof of (60).

We now proceed to show (30). By direct calculation,

$$
\begin{aligned}
\frac{\partial \hat{g}}{\partial y}-\frac{\partial \hat{g}_{*}}{\partial y} & =i \lambda \frac{\partial \hat{\tau}}{\partial y} \cdot \hat{g}-i \lambda \frac{\partial \hat{\tau}_{*}}{\partial y} \cdot \hat{g}_{*} \\
& =i \lambda\left(\frac{\partial \hat{\tau}}{\partial y}-\frac{\partial \hat{\tau}_{*}}{\partial y}\right) \cdot \hat{g}+i \lambda \frac{\partial \hat{\tau}_{*}}{\partial y} \cdot\left(\hat{g}-\hat{g}_{*}\right) .
\end{aligned}
$$

One can check that $\frac{\partial \hat{\tau}}{\partial y}-\frac{\partial \hat{\tau}_{*}}{\partial y}=O\left|\left(t-t_{1}, y\right)\right|^{2}$, then a similar argument as used in the estimate of $R_{1}$ above shows that

$$
\left\|\lambda\left(\frac{\partial \hat{\tau}}{\partial y}-\frac{\partial \hat{\tau}_{*}}{\partial y}\right) \cdot \hat{g}\right\|_{L^{2}(D)}^{2} \lesssim 1 .
$$

Besides, 600 implies that

$$
\left\|\lambda \frac{\partial \hat{\tau}_{*}}{\partial y} \cdot\left(\hat{g}-\hat{g}_{*}\right)\right\|_{L^{2}(D)}^{2} \lesssim \lambda .
$$

Combining these two estimates together, we conclude that

$$
\left\|\frac{\partial \hat{g}}{\partial y}-\frac{\partial \hat{g}_{*}}{\partial y}\right\|_{L^{2}(D)}^{2} \lesssim \lambda .
$$

Similarly, we can show that

$$
\left\|\frac{\partial \hat{g}}{\partial t}-\frac{\partial \hat{g}_{*}}{\partial t}\right\|_{L^{2}(D)}^{2} \lesssim \lambda .
$$

This completes the proof of (30) and hence the lemma.

Proof of Lemma 4.4 Denote $D=\left(3 \epsilon_{1} / 4, t_{1}+\epsilon_{1} / 2\right) \times U\left(x_{1}\right)$ again. We first show (33). Since $x$ is restricted to $V\left(x_{1}\right) \subset \Gamma$, it suffices to show that

$$
\hat{g}^{-}(t, y, \lambda)+\hat{g}(t, y, \lambda)=O(\sqrt{\lambda}) \text { in } H^{1}(D) .
$$

But this is a direct consequence of Lemma 4.2 and the fact that $\hat{g}_{*}^{-}=-\hat{g}_{*}$. 
We now prove (34). By a direct calculation

$$
\begin{aligned}
\frac{\partial g}{\partial \nu}(t, x)= & \frac{\partial}{\partial \nu}\left(a(t) e^{i \lambda \tau(t, x)}\right)=i \lambda g \cdot \frac{\partial \tau}{\partial \nu} \\
= & i \lambda g \cdot\langle\xi(t)+M(t)(x-x(t), \nu(x)\rangle \\
= & i \lambda g \cdot\left\langle\xi\left(t_{1}\right), \nu\left(x_{1}\right)\right\rangle+i \lambda g \cdot\left(\langle\xi(t), \nu(x)\rangle-\left\langle\xi\left(t_{1}\right), \nu\left(x_{1}\right)\right\rangle\right) \\
& +i \lambda g \cdot\langle M(t)(x-x(t), \nu(x)\rangle .
\end{aligned}
$$

Note that in the coordinate $x=F(y)$,

$$
\begin{aligned}
|\langle M(t)(x-x(t)), \nu(x)\rangle| & =O\left(\left|\left(t-t_{1}, y\right)\right|\right), \\
\left|\langle\xi(t), \nu(x)\rangle-\left\langle\xi\left(t_{1}\right), \nu\left(x_{1}\right)\right\rangle\right| & =O\left(\left|\left(t-t_{1}, y\right)\right|\right) .
\end{aligned}
$$

It follows that

$$
\begin{aligned}
\left\|g \cdot\left(\langle\xi(t), \nu(x)\rangle-\left\langle\xi\left(t_{1}\right), \nu\left(x_{1}\right)\right\rangle\right)\right\|_{L^{2}(D)}^{2} & \lesssim \frac{1}{\lambda} \\
\| g \cdot\left\langle M(t)(x-x(t), \nu(x)\rangle \|_{L^{2}(D)}^{2}\right. & \lesssim \frac{1}{\lambda} .
\end{aligned}
$$

Thus

Similarly,

$$
\frac{\partial g}{\partial \nu}(t, x)=i \lambda g \cdot\left\langle\xi\left(t_{1}\right), \nu\left(x_{1}\right)\right\rangle+O(\sqrt{\lambda})
$$

$$
\frac{\partial g^{-}}{\partial \nu}(t, x)=i \lambda g^{-} \cdot\left\langle\xi^{-}\left(t_{1}\right), \nu\left(x_{1}\right)\right\rangle+O(\sqrt{\lambda}) .
$$

Finally, using (33) and the fact that $\left\langle\xi^{-}\left(t_{1}\right), \nu\left(x_{1}\right)\right\rangle=-\left\langle\xi\left(t_{1}\right), \nu\left(x_{1}\right)\right\rangle$, we conclude that (34) holds. This completes the proof of the lemma.

\subsection{An estimate on Gaussian beam interactions.}

Lemma 8.1. Assume that $M_{1}$ and $M_{2}$ are two symmetric positive definite matrices such that $0<c_{0}<M_{1}, M_{2}<c_{1}$, and $N_{1}$ and $N_{2}$ are two symmetric matrices such that $\left\|N_{1}\right\|,\left\|N_{2}\right\| \leq c_{2}$. Let $\delta x, \delta \xi$ be two vectors in $\mathbb{R}^{d}$ and $\lambda \gg 1$. There exists $c_{3}>0$ depending only on $c_{0}, c_{1}$ and $c_{2}$ such that

$$
\left|\int_{\mathbb{R}^{d}} e^{i \lambda \cdot\langle\delta \xi, x\rangle-\lambda x^{T}\left(M_{1}+i \cdot N_{1}\right) x-\lambda(x-\delta x)^{T}\left(M_{2}+i \cdot N_{2}\right)(x-\delta x)}\right| \lesssim \frac{1}{\lambda^{\frac{d}{2}}} e^{-c_{3} \lambda\left(|\delta x|^{2}+|\delta \xi|^{2}\right)} .
$$

Proof. See Lemma 3.7 in [5].

\section{REFERENCES}

[1] G. Alessandrini and J. Sylvester, Stability for a multidimensional inverse spectral theorem, Comm. Partial Differential Equations 15 (1990), no. 5, 711-736, DOI 10.1080/ 03605309908820705. MR 1070844(91i:35198)

[2] S. Bougacha, Jean-Luc Akian, and R. Alexandre, Gaussian beams summation for the wave equation in a convex domain, Commun. Math. Sci. 7 (2009), no. 4, 973-1008. MR2604628 (2011b:35284)

[3] V. I. Arnol'd, Singularity theory: Selected papers, London Mathematical Society Lecture Note Series, vol. 53, Cambridge University Press, Cambridge, 1981. Translated from the Russian; With an introduction by C. T. C. Wall. MR631683 (83d:58016)

[4] V. I. Arnol'd, S. M. Guseĭn-Zade, and A. N. Varchenko, Singularities of differentiable maps, Vol. I: The classification of critical points, caustics and wave fronts, Monographs in Mathematics, vol. 82, Birkhäuser Boston Inc., Boston, MA, 1985. Translated from the Russian by Ian Porteous and Mark Reynolds. MR777682 (86f:58018) 
[5] G. Bao, J. Qian, L. Ying, and H. Zhang, A convergent multiscale Gaussian-beam parametrix for the wave equation, Comm. Partial Differential Equations 38 (2013), no. 1, 92-134, DOI 10.1080/03605302.2012.727130. MR3005548

[6] G. Bao and K. Yun, On the stability of an inverse problem for the wave equation, Inverse Problems 25 (2009), no. 4, 045003, 7, DOI 10.1088/0266-5611/25/4/045003. MR 2482154 (2010a:35270)

[7] M. I. Belishev, An approach to multidimensional inverse problems for the wave equation (Russian), Dokl. Akad. Nauk SSSR 297 (1987), no. 3, 524-527; English transl., Soviet Math. Dokl. 36 (1988), no. 3, 481-484. MR924687(89c:35152)

[8] M. I. Belishev, Boundary control in reconstruction of manifolds and metrics (the BC method), Inverse Problems 13 (1997), no. 5, R1-R45, DOI 10.1088/0266-5611/13/5/002. MR 1474359 (98k:58073)

[9] M. I. Belishev, Recent progress in the boundary control method, Inverse Problems 23 (2007), no. 5, R1-R67, DOI 10.1088/0266-5611/23/5/R01. MR2353313 (2008h:93001)

[10] Michael I. Belishev and Yaroslav V. Kurylev, To the reconstruction of a Riemannian manifold via its spectral data (BC-method), Comm. Partial Differential Equations 17 (1992), no. 5-6, 767-804, DOI 10.1080/03605309208820863. MR1177292(94a:58199)

[11] M. Bellassoued and David Dos S. Ferreira, Stability estimates for the anisotropic wave equation from the Dirichlet-to-Neumann map, Inverse Probl. Imaging 5 (2011), no. 4, 745-773, DOI 10.3934/ipi.2011.5.745. MR2852371

[12] A. Greenleaf and A. Seeger, Fourier integral operators with cusp singularities, Amer. J. Math. 120 (1998), no. 5, 1077-1119. MR 1646055 (99g:58120)

[13] L. Hörmander, The analysis of linear partial differential operators. III, Grundlehren der Mathematischen Wissenschaften [Fundamental Principles of Mathematical Sciences], vol. 274, Springer-Verlag, Berlin, 1985. Pseudodifferential operators. MR781536 (87d:35002a)

[14] V. Isakov, Inverse problems for partial differential equations, 2nd ed., Applied Mathematical Sciences, vol. 127, Springer, New York, 2006. MR2193218(2006h:35279)

[15] A. Katchalov, Y. Kurylev, and Matti Lassas, Inverse boundary spectral problems, Chapman \& Hall/CRC Monographs and Surveys in Pure and Applied Mathematics, vol. 123, Chapman \& Hall/CRC, Boca Raton, FL, 2001. MR1889089(2003e:58045)

[16] I. Lasiecka, J.-L. Lions, and R. Triggiani, Nonhomogeneous boundary value problems for second order hyperbolic operators, J. Math. Pures Appl. (9) 65 (1986), no. 2, 149-192. MR867669 (88c:35092)

[17] John M. Lee, Riemannian manifolds: An introduction to curvature, Graduate Texts in Mathematics, vol. 176, Springer-Verlag, New York, 1997. MR1468735 (98d:53001)

[18] R. Michel, Sur la rigidité imposée par la longueur des géodésiques (French), Invent. Math. 65 (1981/82), no. 1, 71-83, DOI 10.1007/BF01389295. MR636880 (83d:58021)

[19] C. Montalto, Stable determination of a simple metric, a covector field and a potential from the hyperbolic Dirichlet-to-Nuemann map, Comm. PDE., to appear.

[20] J. Qian and L. Ying, Fast multiscale Gaussian wavepacket transforms and multiscale Gaussian beams for the wave equation, Multiscale Model. Simul. 8 (2010), no. 5, 1803-1837, DOI 10.1137/100787313. MR2728710 (2011k:65137)

[21] L. Pestov and G. Uhlmann, The scattering relation and the Dirichlet-to-Neumann map, Recent advances in differential equations and mathematical physics, Contemp. Math., vol. 412, Amer. Math. Soc., Providence, RI, 2006, pp. 249-262, DOI 10.1090/conm/412/07779. MR2259112(2007k:53055)

[22] J. Ralston, Gaussian beams and the propagation of singularities, Studies in partial differential equations, MAA Stud. Math., vol. 23, Math. Assoc. America, Washington, DC, 1982, pp. 206-248. MR716507 (85c:35052)

[23] V. G. Romanov, Inverse problems of mathematical physics, VNU Science Press b.v., Utrecht, 1987. With a foreword by V. G. Yakhno; Translated from the Russian by L. Ya. Yuzina. MR.885902(88b:35203)

[24] V. A. Sharafutdinov, Ray Transform on Riemannian Manifolds, Lecture notes, University of Oulu (1999).

[25] P. Stefanov and G. Uhlmann, Stability estimates for the hyperbolic Dirichlet to Neumann map in anisotropic media, J. Funct. Anal. 154 (1998), no. 2, 330-358, DOI 10.1006/jfan. 1997.3188. MR1612709(99f:35120) 
[26] P. Stefanov and G. Uhlmann, Stability estimates for the X-ray transform of tensor fields and boundary rigidity, Duke Math. J. 123 (2004), no. 3, 445-467, DOI 10.1215/S0012-7094-0412332-2. MR2068966 (2005h:53130)

[27] P. Stefanov and G. Uhlmann, Boundary rigidity and stability for generic simple metrics, J. Amer. Math. Soc. 18 (2005), no. 4, 975-1003, DOI 10.1090/S0894-0347-05-00494-7. MR2163868(2006h:53031)

[28] P. Stefanov and G. Uhlmann, Stable determination of generic simple metrics from the hyperbolic Dirichlet-to-Neumann map, Int. Math. Res. Not. 17 (2005), 1047-1061, DOI 10.1155/IMRN.2005.1047. MR2145709 (2006a:58030)

[29] B. Frigyik, P. Stefanov, and G. Uhlmann, The X-ray transform for a generic family of curves and weights, J. Geom. Anal. 18 (2008), no. 1, 89-108, DOI 10.1007/s12220-007-9007-6. MR2365669(2008j:53128)

[30] P. Stefanov and G. Uhlmann, Integral geometry on tensor fields on a class of non-simple Riemannian manifolds, Amer. J. Math. 130 (2008), no. 1, 239-268, DOI 10.1353/ajm.2008.0003. MR2382148(2009e:53051)

[31] P. Stefanov and G. Uhlmann, Linearizing non-linear inverse problems and an application to inverse backscattering, J. Funct. Anal. 256 (2009), no. 9, 2842-2866, DOI 10.1016/j.jfa.2008.10.017. MR2502425 (2010f:47028)

[32] P. Stefanov and G. Uhlmann, Local lens rigidity with incomplete data for a class of nonsimple Riemannian manifolds, J. Differential Geom. 82 (2009), no. 2, 383-409. MR2520797 (2011d:53081)

[33] Zi Qi Sun, On continuous dependence for an inverse initial-boundary value problem for the wave equation, J. Math. Anal. Appl. 150 (1990), no. 1, 188-204, DOI 10.1016/0022247X(90)90207-V. MR 1059582(91i:35024)

[34] G. Uhlmann, The Cauchy data and the scattering relation, Geometric methods in inverse problems and PDE control, IMA Vol. Math. Appl., vol. 137, Springer, New York, 2004, pp. 263-287, DOI 10.1007/978-1-4684-9375-7_10. MR2169908(2006f:58032)

[35] P. Stefanov and G. Uhlmann, The geodesic X-ray transform with fold caustics, Anal. PDE 5 (2012), no. 2, 219-260, DOI 10.2140/apde.2012.5.219. MR 2970707

[36] H. Zhang, On the stability/sensitivity of recovering velocity fields from boundary measurements, (PhD dissertation), Michigan State University (2013).

Department of Mathematics, Zhejiang University, Hangzhou 310027, China and Department of Mathematics, Michigan State University, East Lansing, Michigan 48824

E-mail address: drbaogang@gmail.com

Department of Mathematics, Michigan State University, East Lansing, Michigan 48824

E-mail address: zh.hai84@gmail.com 